\title{
Diagnostic and prognostic potential of the proteomic profiling of serum-derived extracellular vesicles in prostate cancer
}

\author{
Michele Signore (iD) ${ }^{1}$, Romina Alfonsi $\mathbb{D}^{2}{ }^{2}$, Giulia Federici ${ }^{3}$, Simona Nanni ${ }^{4,5}$, Antonio Addario ${ }^{2}$, Lucia Bertuccini ${ }^{1}$, Aurora Aiello (D) $^{4}$, \\ Anna Laura Di Pace ${ }^{2}$, Isabella Sperduti ${ }^{3}$, Giovanni Muto ${ }^{6,7}$, Alessandro Giacobbe ${ }^{6,7}$, Devis Collura ${ }^{6,7}$, Lidia Brunetto ${ }^{2}$, Giuseppe Simone ${ }^{8}$, \\ Manuela Costantini ${ }^{8}$, Lucio Crinò ${ }^{9}$, Stefania Rossi ${ }^{2}$, Claudio Tabolacci ${ }^{2}$, Marco Diociaiuti ${ }^{10}$, Tania Merlino ${ }^{3}$, Michele Gallucci ${ }^{8,11}$, \\ Steno Sentinelli ${ }^{3}$, Rocco Papalia ${ }^{12}$, Ruggero De Maria ${ }^{4,5,13}$ and Désirée Bonci $\left(\mathbb{D}^{2,3,13}\right.$
}

(c) The Author(s) 2021

Extracellular vesicles (EVs) and their cargo represent an intriguing source of cancer biomarkers for developing robust and sensitive molecular tests by liquid biopsy. Prostate cancer (PCa) is still one of the most frequent and deadly tumor in men and analysis of EVs from biological fluids of PCa patients has proven the feasibility and the unprecedented potential of such an approach. Here, we exploited an antibody-based proteomic technology, i.e. the Reverse-Phase Protein microArrays (RPPA), to measure key antigens and activated signaling in EVs isolated from sera of PCa patients. Notably, we found tumor-specific protein profiles associated with clinical settings as well as candidate markers for EV-based tumor diagnosis. Among others, PD-L1, ERG, Integrin- $\beta 5$, Survivin, TGF- $\beta$, phosphorylated-TSC2 as well as partners of the MAP-kinase and mTOR pathways emerged as differentially expressed endpoints in tumor-derived EVs. In addition, the retrospective analysis of EVs from a 15-year follow-up cohort generated a protein signature with prognostic significance. Our results confirm that serum-derived EV cargo may be exploited to improve the current diagnostic procedures while providing potential prognostic and predictive information. The approach proposed here has been already applied to tumor entities other than PCa, thus proving its value in translational medicine and paving the way to innovative, clinically meaningful tools.

Cell Death and Disease (2021)12:636; https://doi.org/10.1038/s41419-021-03909-z

\section{INTRODUCTION}

Prostate cancer $(\mathrm{PCa})$ is still the second cause of cancer-related male deaths in highly developed countries [1]. A significant fraction of PCa patients arrives at diagnosis with advanced forms, while others retain indolent tumors which will never progress into aggressive stages $[2,3]$. Therefore, an accurate, early diagnosis is likely to improve the outcome and the quality of life of PCa patients while reducing the over-treatment [4].

Extracellular vesicles (EVs) are membrane-enclosed bodies in the nano- to micro-meter scale that are secreted by nearly all cells and shuttle their biological content as a means of cell-to-cell communication $[5,6]$. Tumor cells are now recognized to release more EVs than their normal counterpart and tumor-derived EVs can be easily isolated from bodily fluids [7-10], thus offering an exquisite source in terms of biomarkers and, mechanistically, of cancer treatment strategies [11-13]. The EV sub-population in the range of $30-150 \mathrm{~nm}$ in diameter is referred to as exosomes and has been shown to actively transport DNA, proteins, long and small RNAs [11, 14] as well as small peptides, such as prions [15]. Different from other vesicles, which are generated by random shedding mechanisms or from dying cells by discharge, exosomes drive intra- and inter-tissue cross-talk [16-18], are involved in physiological tissue homeostasis and immune system regulation $[11]$ and in processes $[12,19,20]$ that are often aberrant in tumors [7]. In this regard, PCa is characterized by multiple genomic lesions [21] and several variants seem to be associated with tumor development $[22,23]$. Therefore, the EV cargo in PCa patients could be a promising source of new biomarkers [24] that deserve intense investigation.

The importance of better classifying patients into riskprogression categories has given impetus to the identification of prognostic biomarkers [21]. However, the available diagnostic tools for PCa therapy remain far from satisfying. The application of mass spectrometry-based proteomics has provided insights into the clinical management of PCa [25]. However, the limitations, in terms of sensitivity, throughput and clinical applicability of mass spectrometry, leave margins for other methodologies [25-27].

Here, by using an antibody-based technique, i.e. the ReversePhase Protein microArrays (RPPA), we evaluated the proteomic content of EVs isolated from blood sera of PCa tumors, healthy

\footnotetext{
${ }^{1}$ RPPA Unit, Proteomics Area, Core Facilities, Istituto Superiore di Sanità, Rome, Italy. ${ }^{2}$ Department of Oncology and Molecular Medicine, Istituto Superiore di Sanità, Rome, Italy. ${ }^{3}$ IRCCS, Regina Elena National Cancer Institute, Rome, Italy. ${ }^{4}$ Dipartimento di Medicina e Chirurgia Traslazionale, Università Cattolica del Sacro Cuore Largo F. Vito 1, 00168 Rome, Italy. ${ }^{5}$ Fondazione Policlinico Universitario A. Gemelli IRCCS, Roma, Italy. ${ }^{6}$ Department of Urology, Humanitas University, Turin, Italy. ${ }^{7}$ Department of Urology, S. Giovanni Bosco Hospital, Turin, Italy. ${ }^{8}$ Department of Urology-IRCCS Regina Elena National Cancer Institute of Rome, Rome, Italy. ${ }^{9}$ Department of Oncology, IRST-Meldola, Meldola, Italy. ${ }^{10}$ Department of Rare Diseases, Istituto Superiore di Sanità, Rome, Italy. ${ }^{11}$ Department of Urology, Sapienza University of Rome, Rome, Italy. ${ }^{12}$ Department of Urology Campus Biomedico, Rome, Italy. ${ }^{13}$ These authors contributed equally: Ruggero De Maria, Désirée Bonci. Edited by G. Melino. ${ }^{凶}$ email: desiree.bonci@iss.it
} 
donors, patients with hypertrophic disease, and post-prostatectomy disease-free cases. A retrospective cohort with 15-year follow-up was further included for defining the prognostic value of biomarkers. Our results confirm and expand upon the concept that EVs shuttle cancer-related biomarkers and provide a useful platform for diagnostic as well as prognostic purposes. The combined proteomic and EV-based liquid-biopsy approaches used herein advocate for the use of new, non-invasive cancer monitoring tools in the personalized treatment of PCa.

\section{RESULTS \\ Use of RPPA technology as a proteomic approach to analysis of EV cargo}

We and others have already shown that the RPPA technology is a powerful biomarker discovery platform for the analysis of $\mathrm{EV}$ protein cargo $[28,29]$. Therefore, we decided to leverage on our previous findings to further investigate the analytical performance of a combined RPPA-EV approach. To this end, we first selected suitable cell line models and isolated EVs by differential ultracentrifugation of conditioned culture medium (Fig. S1A). Analysis by Scanning and Transmission Electron Microscope (SEM and TEM, respectively) showed that the majority (90\%) of enriched EVs ranged from 34 to $100 \mathrm{~nm}$ (Fig. 1A, B). RPPA and Western Blotting analyses showed that such isolated EVs expressed transmembrane and cytosolic proteins that are typically enriched in exosomes [30] (Fig. S1B, C). As a specificity test we selected the epithelial cell adhesion molecule (EpCAM) [31, 32] since it is a reference marker in circulating cancer cell capture approaches and is used to search for disseminated tumor cells in bone marrow biopsies [33]. In this regard, we used two lung cancer cell lines, i.e. H1975 and H1299, that are positive and negative for EpCAM, respectively [34, 35] (Fig. S1D). Western blotting and TEM Immunogold labeling confirmed that EVs isolated from H1975 and $\mathrm{H} 1299$ paralleled the expression of EpCAM in their parent cells (Fig. S1E and Fig.1C). In order to test the limit of detection of the RPPA platform, we measured EPCAM in scaling mixtures of H1299and H1975-derived EVs. EpCAM RPPA levels positively correlated with the percentage of H1975-derived EVs and were still detectable over the background down to as low as 3\% of $\mathrm{H} 1975$ EVs (Fig. S1F). Furthermore, we selected HT29, an EpCAM ${ }^{+}$colon cancer line [36] (Fig. S1G) and the metastatic cell lines LNCaP and PC3, positive and negative for EpCAM, respectively (Fig. S2A). Similar to what we found in lung cancer cell lines, we successfully measured EpCAM by RPPA in scaling mixtures of EVs isolated from either HT29 and H1299 (Fig. S1H) or LNCaP and PC3 (Fig. S2B), both showing detectable expression over the background down to $3 \%$ of positive EVs. To expand upon the concept of using RPPA as a sensitive biomarker discovery platform for low-abundance antigens or proteins expressed by low-frequency cancer subclones [37], we studied the expression of two markers, IL-6 and TGF- $\beta$, reportedly shuttled in EVs and over-expressed in advanced cancers $[6,24]$. TGF- $\beta$ and IL- 6 were differentially expressed in PC3 and LNCaP cells (Fig. S2A), and resulted undetectable in EVs by Western blotting (data not shown). Conversely, RPPA analysis of LNCaP- and PC-derived EVs, mixed in scaling percentages, showed that TGF- $\beta$ and IL- 6 expression in EVs mirror the pattern found in parent cells (Fig. S2C, D). Notably, when measuring TGF- $\beta$ by RPPA in EVs isolated from PC3 cells along with a cytokine reference standard, we were able to detect down to as low as $7 \mathrm{pg} / \mathrm{mL}$ of TGF- $\beta$ in PC3-derived EVs (Fig. S2D).

Besides merely testing the analytical performance of RPPA, we were particularly interested in measuring post-translational modifications, e.g. phosphorylation, of proteins shuttled by EVs. To this end, we evaluated the expression of the epithelial-growth factor receptor (EGFR) and one of its phosphorylated forms (EGFR_pY1068) in A431, an epithelial squamous carcinoma cell line, and in human PCa-derived activated fibroblasts (CAFs).
Indeed, EGFR is expressed at high levels by A431 $\left(1-2 \times 10^{6}\right.$ receptors per cell $[38,39])$ while being low to undetectable in fibroblasts [40]. EVs were isolated from culture media and mixed in scaling percentages for RPPA analysis. As expected, the levels of EGFR were proportional to the percentage of A431 in each mixture point (Fig. S2E) while those of PD-L1, reportedly expressed by both A431 and CAFs [41, 42], did not follow a dilution curve (Fig. 2E). In line with the expression of its total content counterpart, RPPA levels of phosphorylated EGFR (EGFR_pY1068) positively correlated with the fraction of A431 in mixed A431/CAFs EVs (Fig. S2F). Driven by these results, we sought to quantify the levels of EGFR_pY1068 in scaling mixtures of A431 and CAFs as well as of their isolated EVs using a reference curve of a synthetic EGFR_pY1068 peptide, ranging from the nanogram down to the picogram scale. Interestingly, although cell-derived extracts displayed significantly higher amounts, RPPA expression of EGFR_pY1068 was still detectable below the picogram range in sample mixtures with increasing fractions of A431-derived EVs (Fig. 1D). Even though the sensitivity and specificity may depend on both the individual antibody performance and antigen relative expression, our data soundly suggest that the proposed approach is feasible and robust.

\section{Validation of RPPA results by an ELISA-based assay}

In order to validate and integrate RPPA with orthogonal approaches, we set up a homemade ELISA (enzyme-linked immunosorbent assay), hereafter referred to as ELEXO. In such an assay, EVs are directly coated to the plastic plate and are subsequently probed for the expression of different surface markers by using primary and HRP-conjugated secondary antibodies followed by colorimetric detection (Fig. 2A). Thus, we took advantage of ELEXO to measure EpCAM and the exosomal marker CD81 on EVs isolated from H1975 and H1299. Similar to our previous RPPA and Western blot analyses, EpCAM levels were significantly detectable over the background only in H1975-derived EVs (Fig. 2B). EVs isolated from HT29 and SW480 colorectal cancer cell lines express CD81 and display differential levels of EpCAM by Western Blotting (Fig. S3A) as well as by ELEXO (Fig. S3B). Subsequently, we focused on the clinically relevant antigen PD-L1 [43] and, in order to test the antibody specificity, we transduced 293T cells with either empty (TWEEN) or PD-L1 gene (PD-L1) lentiviral vectors, respectively. We then isolated EVs from relative culture media and successfully measured PD-L1 by ELEXO only on those deriving from PDL-1transduced cells (Fig. S3C). In addition, since both H1975 and H1299 cells express PD-L1 (Fig. S3D) we used ELEXO to confirm the presence of PD-L1 on their secreted EVs (Fig. S3E). Similar results were obtained by RPPA analysis of PD-L1 levels on a series of matched cells and cell-derived EVs including H1975 and H1299 samples (Fig. S3F).

Encouraged by these results, we envisioned an EV-based liquid-biopsy approach [44] built on RPPA analysis of EVs isolated from cancer patients' serum samples. The majority of isolated vesicles (85\%) showed sizes ranging between 34 and $100 \mathrm{~nm}$ as by SEM and TEM analyses (Fig. 2C-E and Fig. S4A). Analysis of typical exosome markers [45-47] confirmed their expression in EVs derived from sera of a small set of healthy donors, hypertrophic disease and PCa samples by Western blotting (Fig. S4B). Similarly, we measured exosomal proteins by RPPA in EVs isolated from a set of 12 PCa samples (Fig. S4C). Then we used ELEXO to evaluate EpCAM levels in EVs isolated from the serum of 7 colon adenocarcinoma (CRC) samples (Table S1). Our results showed that EpCAM is not only expressed by colon tumors [31, 32], but also a potential biomarker for CRC-derived EVs (Fig. 2F). Since PD-L1 is a predictor biomarker of immunotherapy response in advanced Non-Small-Cell lung cancer (NSCLC) [48], we sought to apply ELEXO to detect PD-L1 in serum EVs isolated from 6 NSCLC samples (Table S1, Fig. 2G), thus 
A

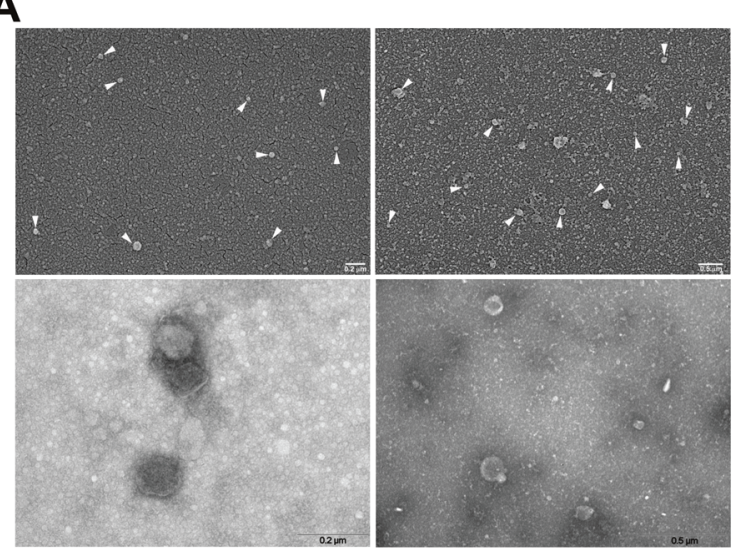

C

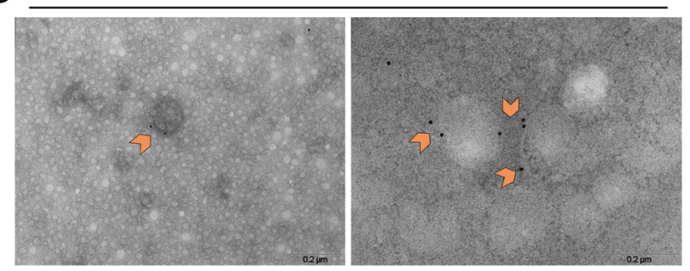

B
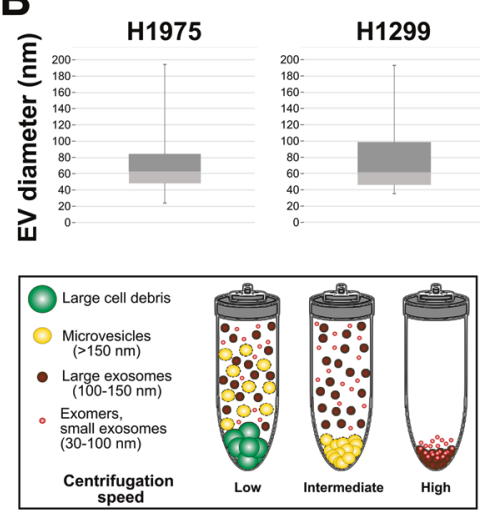

H1299

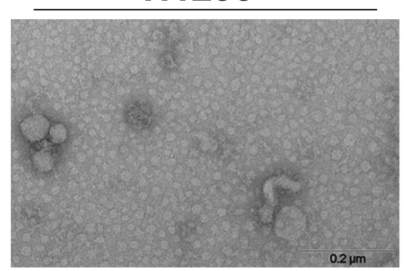

D


Fig. 1 Isolation and RPPA testing of cell line-derived EVs. A Representative images of EVs purified from conditioned medium of H1975 and H1299 cells by Scanning and Transmission Electron Microscopy (SEM and TEM) analyses, upper and lower panels, respectively. The images shown are representative of all cell lines used in the article. B Boxplots representative of H1975 and H1299 EVs size distribution evaluated by SEM analysis. The lower schematic representation exemplifies the size distribution of EVs. C EpCAM antigen evaluated in EVs purified from cultures of H1975 and H1299 by Immuno-Electron Microscopy (IEM). D RPPA measurement of absolute amounts of EGFR_pY1068 in A431 and fibroblasts. Whole cell and EV lysates were first diluted to a total protein concentration of $0.5 \mathrm{mg} / \mathrm{ml}$ and then printed as mixtures with the indicated decreasing and increasing fractions of A431 and fibroblasts, respectively. A dilution curve of EGFR_pY1068 synthetic peptide in the plotted range, was printed along with cell line and EV samples and provided a reference curve (lower-left panel) to predict absolute concentrations of EGFR_pY1068 in A431 and fibroblast mixtures (upper panel). Data in the left side of the upper panel represent a magnification of the low picogram range from the adjacent (right) scatterplot. Main points represent the mean of technical replicates $(n=3$, empty symbols behind each main-colored point). The reference curve in the lower panel has been used to predict EGFR_pY1068 absolute levels in samples via simple linear regression (red line) of log10-converted triplicates of normalized RPPA levels from the EGFR_pY1068 synthetic peptide dilution curve. Adjusted $r$ squared, regression formula and p-value are shown inside the reference curve plot.

confirming the clinical applicability of ELEXO. Parallel to the above experimental design on cell line models, we tested the possibility to use RPPA to measure, via a reference dilution curve, the levels of EGFR_pY1068 in EVs isolated from the serum of primary NSCLC and CRC samples $(n=35)$ (Table S1). Our results show that, although not quantifiable, EGFR_pY1068 is still detectable above the background even below the picogram level (Fig. $2 \mathrm{H}$ ) suggesting the opportunity for accurate measurement of phosphorylated and non-phosphorylated analytes in serum-derived EVs. 
A

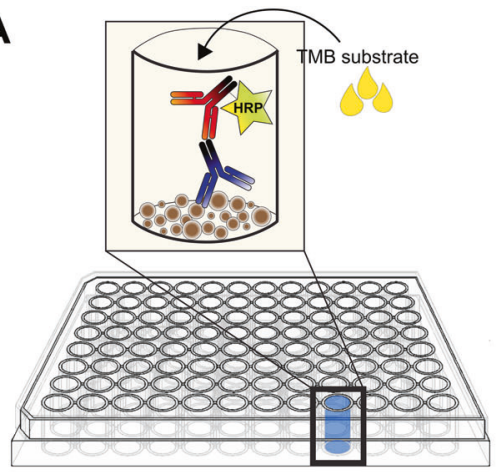

B

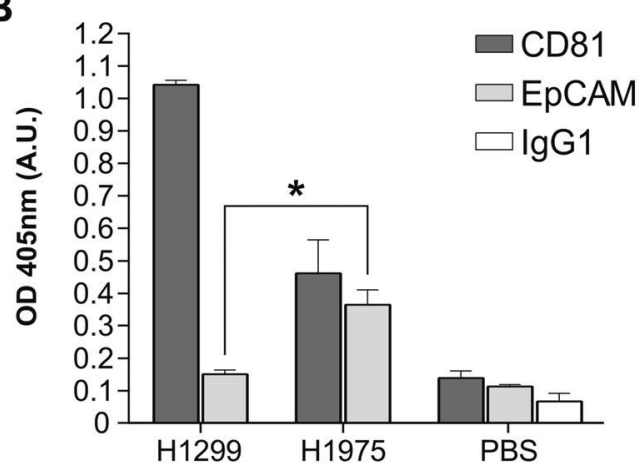

C

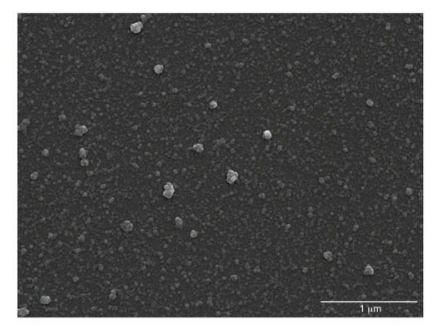

D

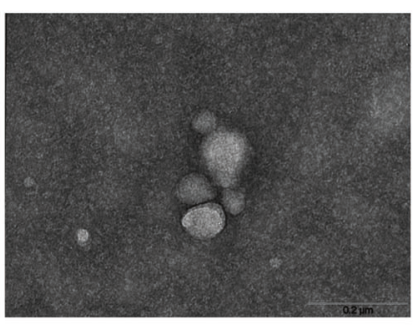

E

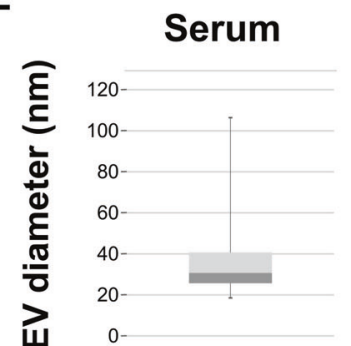

F
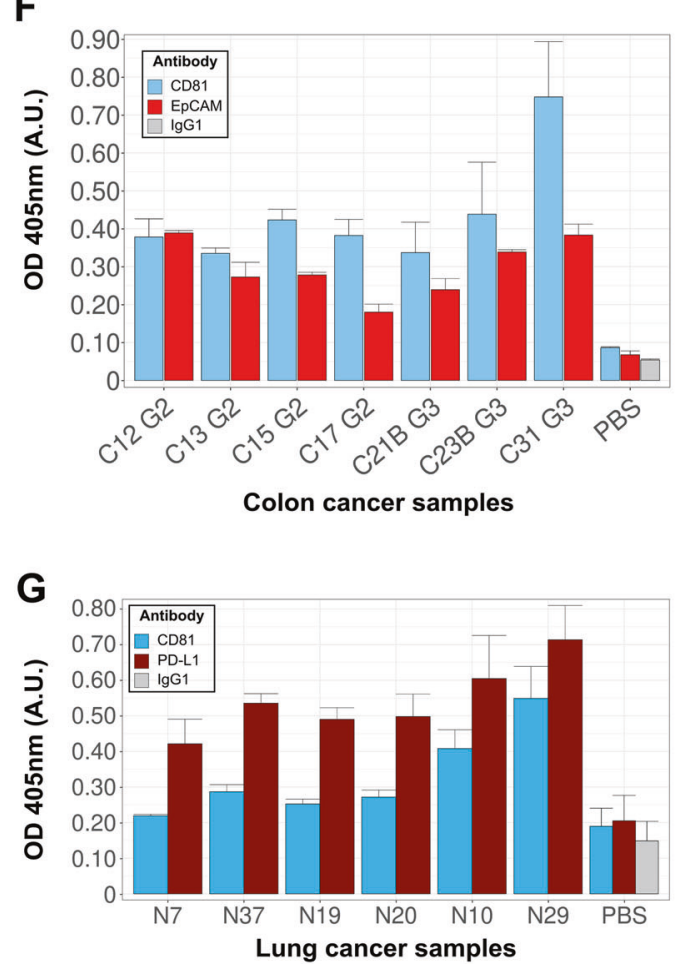

H
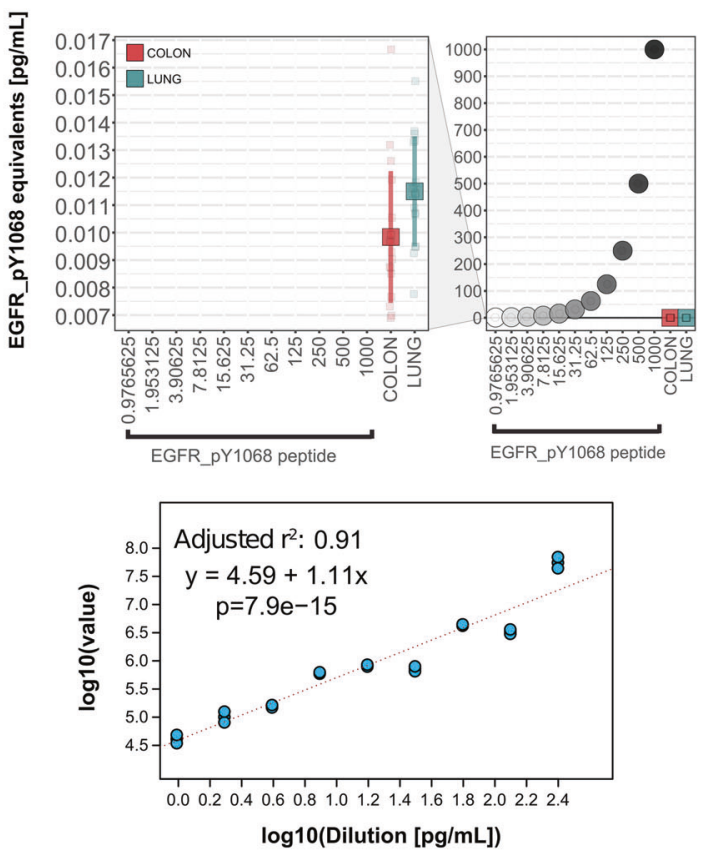

Fig. 2 Orthogonal validation of RPPA results on serum-derived EVs. A Representative scheme of our homemade ELISA test, i.e. ELEXO, including EV-coated microtiter plate, primary antibody, HRP-conjugated secondary antibody and colorimetric reaction with TMB substrate. B H1975 and H1299 EVs analyzed by ELEXO assay. IgG isotype and Phosphate-Buffered Saline (PBS) were used as internal controls. Data are reported as mean and SD $(n=3)$ of arbitrary units of O.D. (Optical Density) at the specified wavelength $(\mathrm{nm})\left({ }^{*} p=0.02\right)$. C, D Representative images of SEM (C) and TEM (D) analysis of EVs isolated from the sera of prostate cancer patients. Images are representative of pivotal and training cohort PCas. E Box plot representative of serum EV size distribution evaluated by SEM analysis. F, G ELEXO measurement of EpCAM (F) and PD-L1 (G) antigens in EVs isolated from colon and lung cancer patients, respectively. CD81 has been used as endogenous EV control. Data are reported as mean and SD $(n=3)$ of arbitrary units of O.D. (Optical Density) at the specified wavelength (nm). Internal reference controls were reported as IgG, CD-81, EpCAM, or PD-L1 antibody in PBS condition. H Absolute RPPA quantification of EGFR_pY1068 synthetic peptide in dilution curve along with isolated EVs from sera of colon and lung cancer patients. The expression levels in EVs fall below the lowest EGFR_pY1068 peptide dilution point and their scale is magnified in the left panel of the plot. The reference curve in the lower panel was obtained as in Fig. 1D to predict absolute EGFR_pY1068 RPPA levels in EV samples. 
A

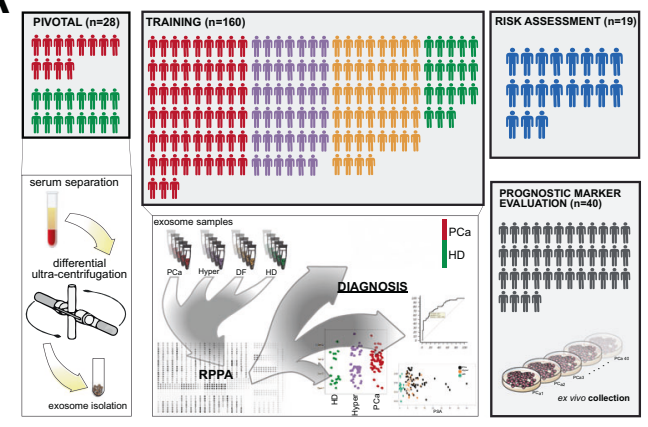

$\square$ Primary Tumors (PCa) $\square$ Hypertrophic (Hyper) $\square$ Post-prostatectomy (DF) $\square$ Healthy donors (HD) $\square$ (non-)Recurrents (RnR) $\square \underset{\text { Castr. resistant (CR) }}{\text { Biocher recerent }} \square$ ex-vivo

C

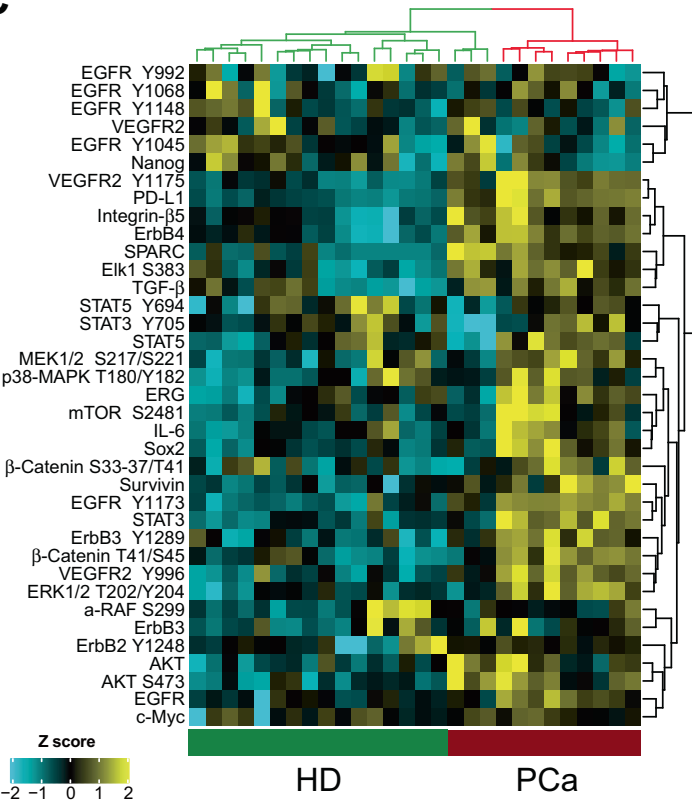

E

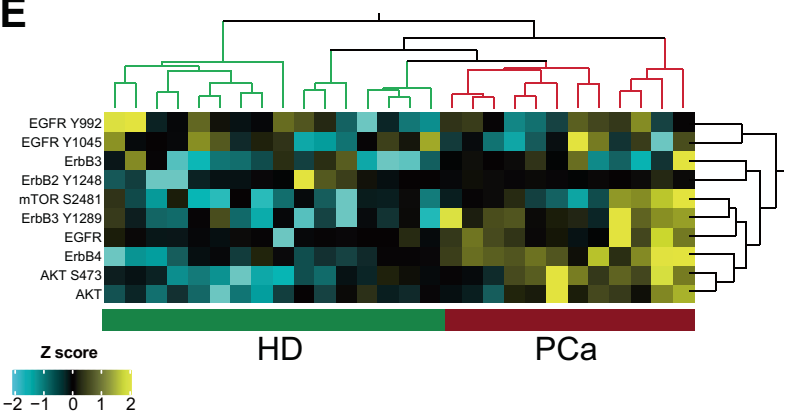

B

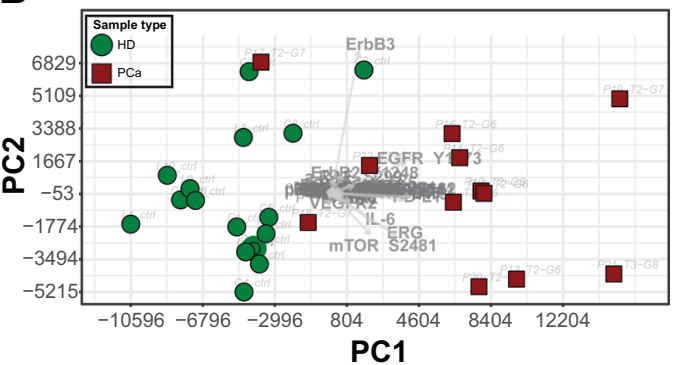

D

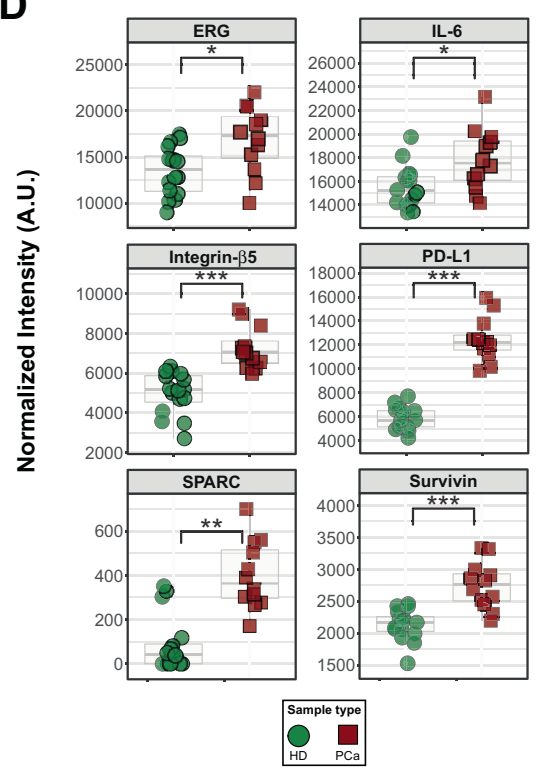

$\mathbf{F}$

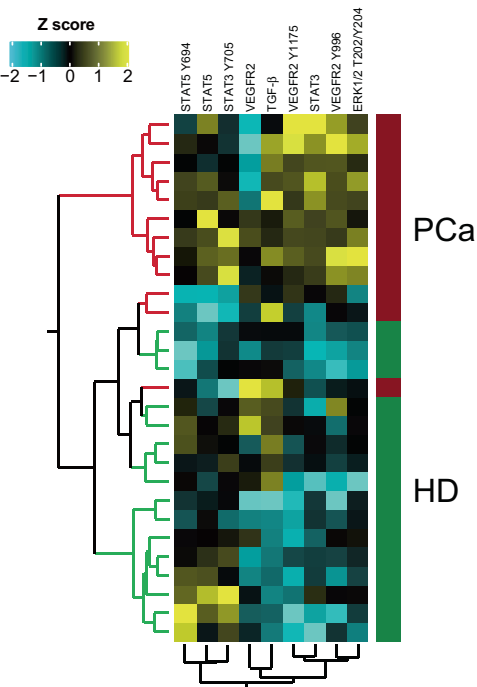

\section{Pivotal analysis on a cohort of PCa patients}

Inspired by the results obtained by RPPA and ELEXO on EVs derived from cell lines and cancer patients' sera, we designed a series of study sets tailored to PCa and graphically depicted in Fig. 3A. First, we conducted a RPPA experiment on the restricted set of patients referred to as 'pivotal cohort', composed only of PCa patients and healthy individuals (HD) (Fig. 3A). Surprisingly, we found that analysis of serum-derived EVs using as low as thirty-seven proteins, selected by their relevance in oncological processes (Fig. S4D), is per se sufficient to infer sample origin (Fig. 3B and Fig. S5A). Hierarchical clustering of RPPA data confirmed that such EV-based proteomic analysis differentiates PCa from HD (Fig. $3 \mathrm{C}$ ). In particular, ERG (ETS-related gene), PD-L1, Survivin, Integrin- $\beta 5$, IL-6 and SPARC were significantly altered in tumoral EVs (Fig. 3D). Prospectively, RPPA analysis of key EV (phospho-) proteins such as those involved in the EGFR, mechanistic target of rapamycin (mTOR) and vascular endothelial growth factor receptor (VEGFR) pathways, allow cancer- and patient-specific adoption of treatment strategies (Fig. 3E, F). 
Fig. 3 RPPA study sets and results on the pivotal cohort. A Schematic representation of the experimental study design. The cohorts assayed comprise a (i) pivotal group of primary prostate cancer (PCa) and healthy donor (HD) samples that have been utilized for experimental setup, i.e. EV isolation and RPPA sensitivity tests, (ii) training cohort including PCa, hypertrophic (Hyper), post-prostatectomy-disease-free (DF) cases as well as healthy donor (HD) EV samples and used to confirm and expand upon the RPPA analysis of the pivotal cohort in search of diagnostic markers, iii) a set of two independent cohorts of PCa samples used for risk assessment and prognostic marker evaluation, respectively. The risk assessment cohort comprises samples with 15-year documented follow-up (recurrent and non-recurrent). The prognostic marker evaluation cohort is composed of forty primary cell lines established from patients with bad and good documented prognosis [66]. B Principal component analysis (PCA) of 37 RPPA endpoints measured in EV samples from the pivotal cohort (16 healthy donor, HD, and 12 tumors, PCa). PCA algorithm employed the covariance matrix obtained from normalized RPPA intensity values. Scores (i.e. samples) are represented as dots (HD, green) and squares (PCa, red) while loadings (i.e. RPPA antibodies) are overlaid and pointed by gray arrows. C Two-way unsupervised hierarchical clustering of the same dataset as in (B). Normalized RPPA intensity values were pre-standardized ( $Z$ score) and the color intensity scale indicates high (yellow), average (black) and low (cyan) relative expression. D Scatterplots of selected statistically significant RPPA endpoints, resulting from comparison of HD- and PCa-derived EVs. Statistical significance by Student's $t$ test is reported on each plot and coded with asterisk(s) based on the level of significance $\left.{ }^{*} p<=0.05,{ }^{* *} p<=0.01,{ }^{* * *} p<=0.001\right)$. E, F Two-way unsupervised hierarchical clustering on selected panels of significant RPPA endpoints $(p<=0.05)$ associated with growth factor receptor signaling and angiogenesis (respectively E, F). Statistical significance was calculated by comparing normalized RPPA intensity values of HD and PCa, as in (D). Hierarchical clustering was performed on standardized RPPA data as in (C) and Z scores are color-coded as high (yellow), average (black) and low (cyan).

It is worth noting that ERG protein over-expression is under investigation as surveyor genomic aberration for the application of novel diagnostic tools in PCa [49]. Along similar lines, PD-L1, Survivin and Integrin- $\beta 5$ have already been found in vesicles isolated from cancer patients [48-51], SPARC, STAT3 and activated EGFR (EGFR_pY1173), have been described as predictors of response to targeted therapy [52-54] while IL-6 is known to promote prostate tumorigenesis and progression to aggressiveness [52, 55]. Indeed, soluble serum protein components such as IL-6 may eventually contaminate the EV cargo following the differential ultracentrifugation steps [56]. Therefore, we devised an ad hoc experimental design and analyzed a cytokine panel by Luminex technology on two paired samples obtained from the same preparation of serum-derived tumoral EVs. In details, we collected $100 \mu \mathrm{g}$ of EVs left un-lysed in the supernatant from the last-ultracentrifugation step (SN), likely containing contaminant proteins, along with an identical total protein content $(100 \mu \mathrm{g})$ from EVs lysed in RIPA buffer (lysed- RIPA) and likely containing residual, membrane-bound contaminants as well as proteins from the EV cargo. While other cytokines were still present in the both samples, IL-6 resulted higher in the RIPA-lysed preparation (Fig. S4E, F). These results suggest that, although soluble serum proteins may still be an undesired contaminant of the EV preparation by differential ultracentrifugation, in our hands the IL- 6 levels measured by RPPA are likely to derive mainly from the EV cargo.

In search of further confirmation on the importance of the differentially expressed targets emerging from our RPPA analysis, we evaluated the levels of mRNAs corresponding to selected RPPA antigens (Fig. 3D) in publicly available PCa datasets (https://www.cbioportal.org/ [21] and http://gepia. cancer-pku.cn/index.html [57]). Although tissue mRNA expression represents an analytical and molecular scenario potentially different from released EVs, still we found that half of our RPPA PCa markers were upregulated at the mRNA level when comparing tumor tissues with their normal adjacent counterpart (Fig. S5B, C). Overall our results and, although partial, such additional in silico data, strengthen the idea of integrating the available molecular profiling with proteomic analysis of EVs for identifying tumor-specific marker and druggable targets for improvement of patient's outcome.

\section{Evaluation of EV protein cargo as a diagnostic tool in PCa}

In order to expand upon and confirm our preliminary experimental evidence on the pivotal cohort, we analyzed a larger patient set, hereafter referred to as the "training cohort" (Fig. 3A) and composed of: (i) healthy donors (HD); ii) over twelve months disease-free patients (DF); iii) hypertrophic cases (Hyper) and iv) tumors (PCa) (Table S1). Following enrichment by differential ultracentrifugation, EVs isolated from PCa sera showed higher total protein content if compared to $\mathrm{HD}$ and DF groups while Hyper showed levels close to those of PCa patients (Fig. S5D). The panel of RPPA endpoints analyzed in the training was enlarged with respect to the pivotal cohort and comprised total and phosphorylated antigens involved in diverse hallmark cancer signaling pathways (Fig. S6). To better define key disease-specific markers, we compared PCa to each of the other groups (i.e. either Hyper or DF or HD) and found significantly different antigens exclusive of individual comparison sets as well as those shared between all the aforementioned comparisons (Fig. 4A, Tables S24). Subsequently, we searched for diagnostic candidates based on their individual performance in the $\mathrm{ROC}$ curve analysis of $\mathrm{PCa}$ versus controls (grouped HD and Hyper). Intriguingly, phospho-cMyc_T58/S62 and phospho-TSC2_Y1571 emerged as the best candidates and, although their combination resulted in a modest gain of diagnostic power, it might still be of clinical relevance in the context of PCa (Fig. 4B). Graphical representation of the data showed that, compared to controls, at least half of the PCa had lower phospho-c-Myc_T58/S62 combined to higher phosphoTSC2_Y1571 (Fig. 4C). Notably, these results held true even when comparing $\mathrm{PCa}$ to the HD group alone (Fig. S7A). Among key endpoints reaching statistical significance in all comparison sets (i.e. PCa versus either Hyper or DF or HD), we found components and interactors of the MAP-kinase/mTOR pathway [58] such as TGF- $\beta$, phospho- $\beta$-Catenin_T41/S45 ( $\beta$-Catenin_pT41/pS45) and phospho-C-Myc_T58/S62 itself (Fig. $4 \mathrm{~A}$ and Tables S2-4, gray boxes), eventually underlying a common aberrant pathway route (Fig. S7B). In addition, several RPPA endpoints emerged as significantly altered or shared when including all available paired comparison sets (i.e. each group versus each of the others) (Fig. S7C).

Interestingly, for a subset of the proteins analyzed in the training, we reproduced the differential expression pattern obtained in the pivotal cohort. In detail, high levels of SPARC were significantly associated with PCas while an increase in PD-L1 and Survivin characterized both inflammatory and neoplastic disease (Fig. S7D). Although ERG did not reach statistical significance in the training cohort, a subgroup of PCa showed elevated (above the 65th percentile) ERG expression and likely associated with high-risk patients as well as with evidence of progression within 3 years (Fig. S8A). Conversely, while in the pivotal cohort IL-6 emerged as a differentially expressed antigen (PCa versus HD), in the training cohort the levels of IL-6 showed a modest, not significant trend of correlation with increasing pathological Tumor-Node-Metastasis (pTNM) staging (Fig. S8B). Intrigued by this result, we sought to correlate key proteomic EV markers with standard clinical parameters such as prostatespecific antigen (PSA) levels and pTNM. While PSA levels in PCa 

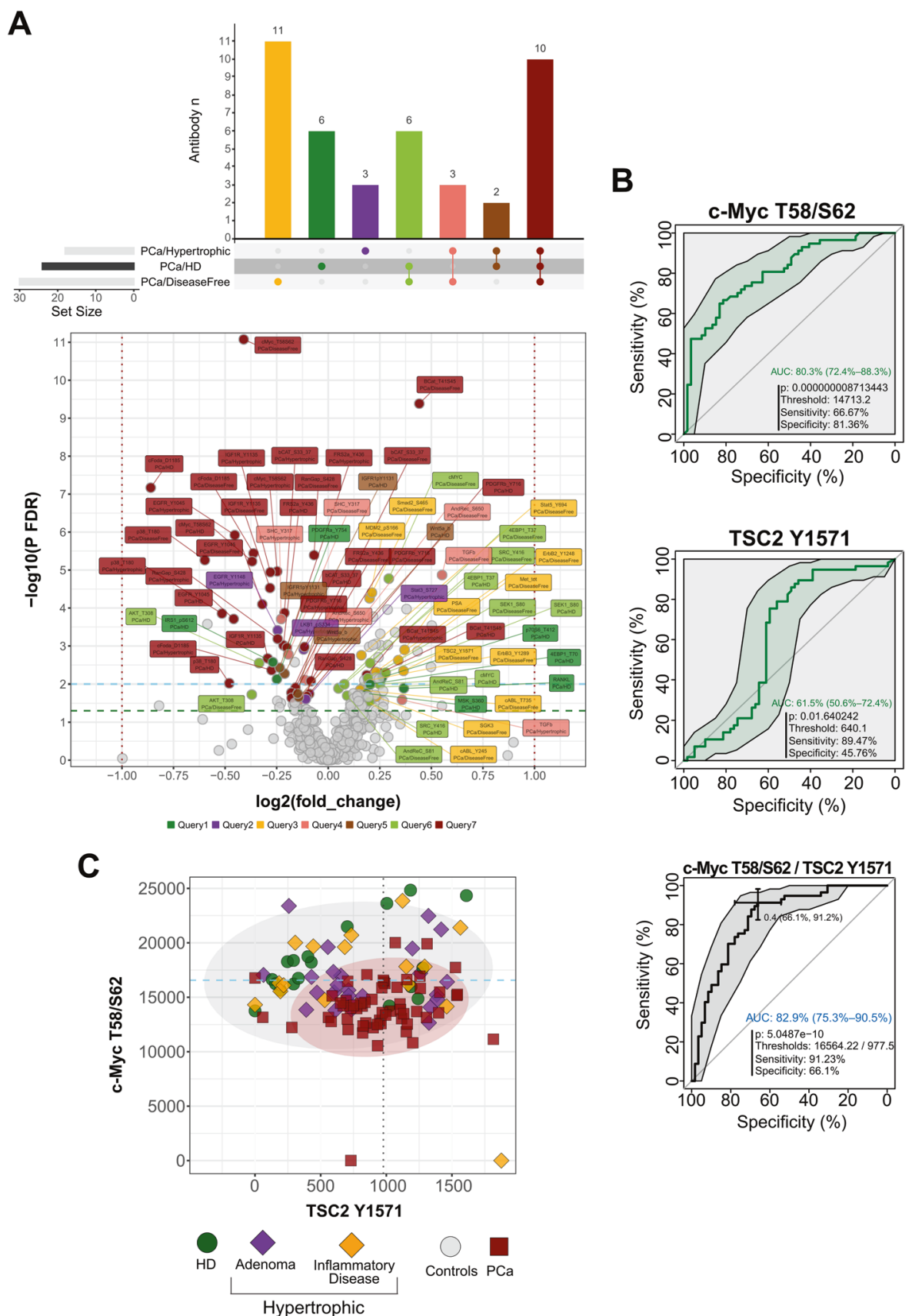

Fig. 4 Group comparisons and biomarker analysis on the training cohort. A Combined Up Set [94] and volcano plots, with color-coded annotations of diverse RPPA endpoint sets that characterize specific binary comparisons of sample groups. The vertical histogram (upper panel) reports the relative frequency of unique (single dots) and shared (connected dots) significant RPPA antibodies resulting from three specific sets of statistical comparisons, namely PCa versus Hypertrofic, HD and Post-prostatectomy disease free (DF), respectively. The volcano plot in the lower panel shows fold-changes (log2) versus significance $[-\log 10(p$-value)] for all analyzed comparison sets (i.e. PCa versus Hypertrofic or HD or disease-free, respectively). The color-coding ('queries') of RPPA antibody labels in the volcano plot matches the corresponding colors sets in the main frequency histogram. The horizontal histogram adjacent to comparison sets (left-bottom part of the upper panel) shows the absolute frequency of statistically significant RPPA antibodies obtained for each individual set. B Univariate ROC curve analysis of two selected, significant candidate markers resulting from statistical comparison of controls (grouped HD plus Hypertrophic diseases) and tumors (upper plots). The diagnostic performance of combined top-scoring candidates was further assessed by ROC curve analysis (bottom plot). All plots report the AUC value along with the $95 \%$ confidence interval as well as p, optimal cut-off, sensitivity and specificity values. C Bivariate plot of best candidates (c-Myc T58/S62 and TSC2 Y1571) normalized RPPA intensity values in the analyzed cohort comprising tumors (PCa) and control samples, i.e. HD and Hypertrophic diseases, the latter subdivided into Adenoma and Inflammatory diseases, being the category 'others' referred to grouped non-neoplastic samples (controls). The ellipses represent the probability of distribution ( $95 \%$ confidence under normality assumption) for the two main comparison groups, namely PCa and others. 


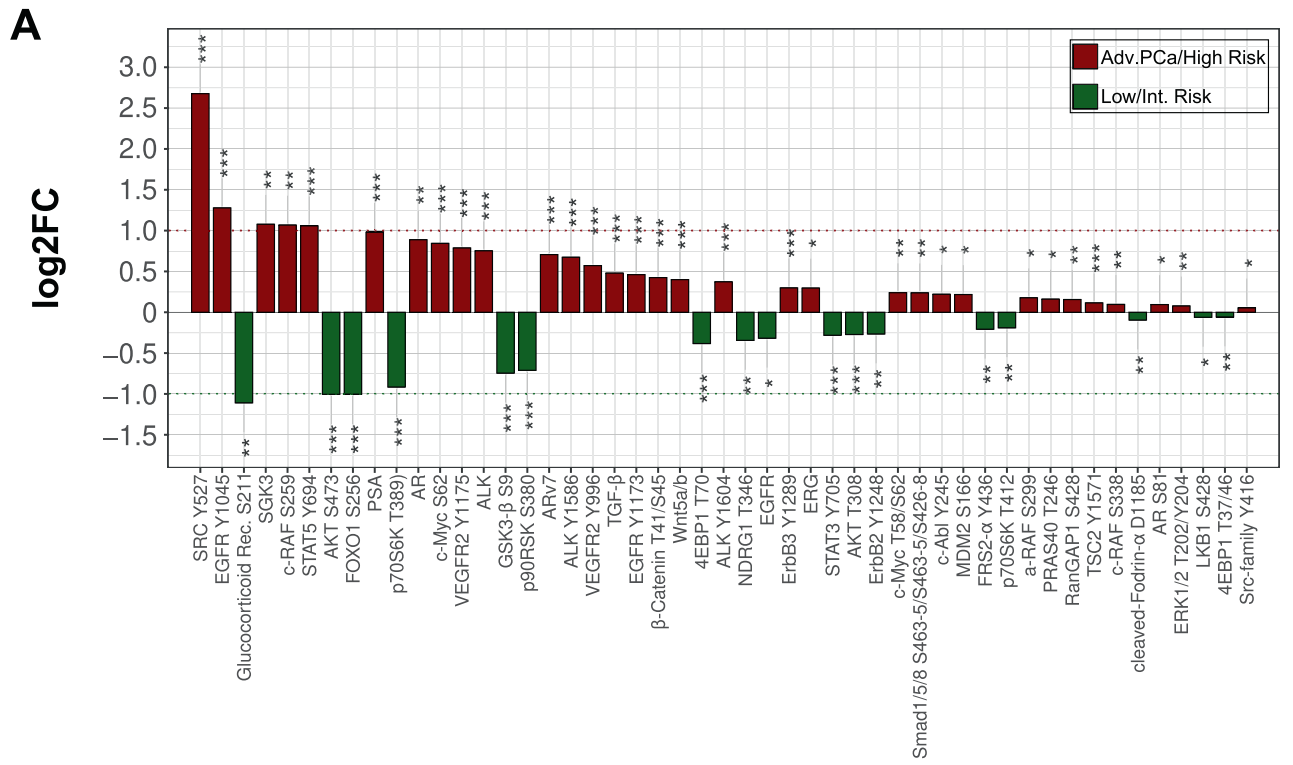

B

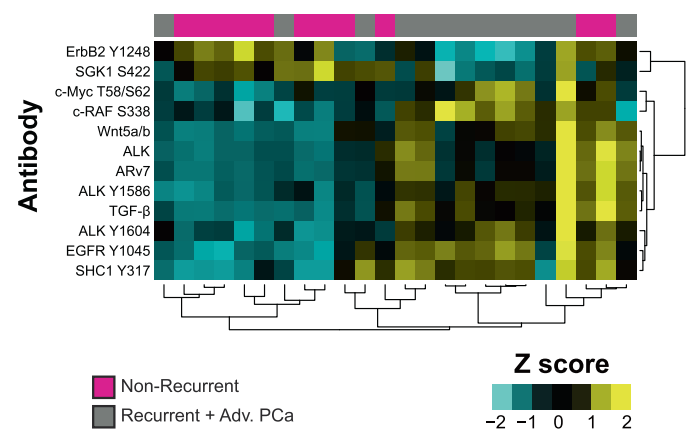

C

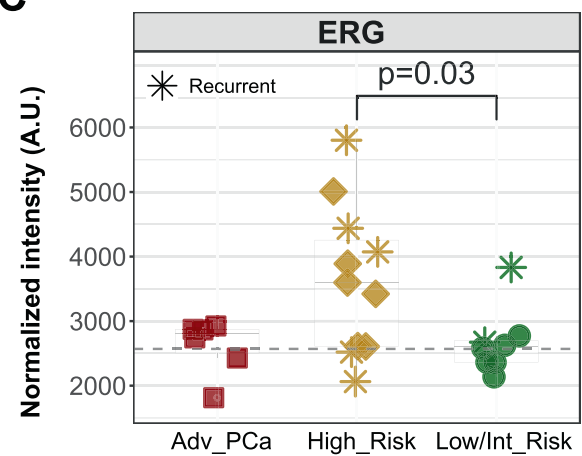

Fig. 5 Analysis of EV-based prognostic RPPA biomarkers. A Bar chart of log2 fold-change (log2FC) of grouped high-risk and advanced PCa (red) versus low-/intermediate-risk (green) patients for all significantly different RPPA endpoints analyzed by Wilcoxon rank-sum test. Statistical significance reported for each bar is coded with asterisk(s) based on the level of significance $\left({ }^{*} p<=0.05,{ }^{* *} p<=0.01,{ }^{* * *} p<=0.001\right)$. B Twoway unsupervised hierarchical clustering of the 13 significantly different $(p<=0.05)$ RPPA endpoints emerging from statistical comparison of non-recurrent (pink, $n=11$ ) and grouped recurrent and advanced tumor (gray, $n=13$ ) EV samples. One sample was excluded due to lacking follow-up information. C Scatterplots of ERG expression resulting from comparison of low/intermediate-, high-risk and advanced (Adv. PCa) tumors by RPPA analysis. Recurrent patients were indicated by an asterisk $(*)$. The plots represent distribution of RPPA intensity values, and the line indicates an arbitrary baseline. Statistical comparisons were performed as described in the methods section.

and benign prostatic hyperplasia largely overlap at a range of $4-10 \mathrm{ng} / \mathrm{ml}$ [59], in such a gray zone we found, for a few of the analyzed markers, that a fraction of PCa samples was distinguishable from hypertrophic diseases in terms of RPPA expression levels (Fig. S8C, D). Furthermore, a restricted number of RPPA endpoints displayed a correlation trend with pTNM as well as with pTNM and PSA (Fig. S8E).

Overall, the RPPA analysis of EV protein content revealed a set of cancer-specific biomarkers and demonstrated that a targeted liquid-biopsy approach allows discrimination of patients from healthy individuals. Our data suggest that combination of proteomic analysis of EVs with conventional clinical parameters may prove useful for early cancer detection.

\section{Targeted analysis of EVs proteome as prognostic and monitoring tool in PCa}

Due to the relatively high life expectancy of diagnosed patients, the PCa risk management is a fundamental research field [60]. To identify biomarkers that are predictive of the risk of progression, we studied a retrospective cohort (Risk Assessment, Fig. 3A, Table S5) comprehensive of six advanced castration-resistant (CR) patients as reference controls. Serum samples were collected before surgery for the 19 primary tumor cases and during the recurrence phase for the advanced CR patients. Isolated EVs underwent RPPA profiling and, interestingly, high-risk and advanced tumors showed shared expression of several antigens (Fig. S9A). Therefore, in order to find a common signature, we grouped high-risk patients with advanced tumors and compared them to low/intermediate-risk cases (Fig. 5A). Moreover, we compared high-risk patients alone to either advanced $\mathrm{PCa}$ or low/intermediate-risk cases (Fig. S9B, C). The differentially expressed antigens characterizing the high-risk and advanced PCa patients and comprising components of the cell cycle, PI3K, WNT, and MAPK pathways, likely reflect the higher frequency of genomic alterations found in aggressive forms [21]. Indeed, mining of publicly available PCa tissue dataset [21] confirmed an increased number of mutations in advanced forms, both at the pathway and at the gene level, if looking at the genes corresponding to the differentially expressed RPPA endpoints (Fig. S9D, E). Then, we investigated the association between RPPA antigen levels and the frequency of recurrence in the retrospective, i.e. Risk Assessment, cohort (Fig. 3A). Seven out of 19 
primary cases-with the exclusion of one sample lacking a documented follow-up-developed metastases over a 15-year time frame (Table S5). Intriguingly, heatmap with significantly different RPPA endpoints showed that the non-recurrent cases converged into a separate cluster when compared to grouped recurrent cases and advanced tumors (Fig. 5B), advocating for potential prognostic implications of such an analytical approach. Along these lines and similar to the strategy used to individuate the best diagnostic candidates in the training cohort, we performed statistical comparisons and ROC curves and found RPPA targets that may allow for EV-based discrimination of recurrent cases and advanced forms. In particular, phospho-cMyc_T58/S62, phospho-SHC_Y317 and Wnt5a/b as well as phospho-c-RAF_S338 and TGF- $\beta$ reached statistical significance and emerged as optimal prognostic candidates (Fig. S10A and Fig. S10C, individual antigens). Advanced PCa has the propensity to metastasize to bone and, notably, TGF- $\beta$ mRNA over-expression is associated with tumor invasion and spreading to bones [61]. Therefore, we investigated a publicly available dataset (GEO GSE74685 [61]) and found that, consistently with our results obtained in EVs (Fig. S10C), the levels of tissue mRNAs for genes corresponding to RPPA candidates, i.e. c-Myc, SHC-1, Wnt5a, cRAF-1 as well as TGF- $\beta$, were increased in bone metastases as compared to all other metastatic sites (Fig. S10D). We then asked whether any combination of these five antigens could gain prognostic and predictive value over individual ROC curves. Of note, the concomitant random expression of almost three out of the five candidate RPPA endpoints at levels beyond their respective cut-offs, was able to produce a significantly improved prognostic signature (Fig. S10C, Score). Again, we sought to corroborate our results by analysis of mRNA levels of the corresponding genes from publicly available gene expression datasets, namely [21] and [62]. When comparing metastatic and primary PCa samples, only $W n t 5 a / b$ was significantly and congruently upregulated in both datasets (Fig. S10E), suggesting that our data and EV-based approach provide complementary information to standard tissue transcriptomic characterization. Interestingly and similarly to what we have shown here for the pivotal and training sets and others have already shown [63, 64], we observed ERG protein expression beyond the calculated cut-off (i.e. over-expressed) in high-risk patients (Fig. 5A) and in low- and high-risk categories (Fig. 5C). Of note, elevated ERG protein expression in recurrent patients (Fig. 5A) mirrored gene expression data from two public datasets (GEO GSE32269 [62] and GSE74685 [61], Fig. S10F, G, respectively), whereby ERG over-expression has been shown to parallel the genomic fusion and to contribute to PCa progression by WNT and MAPK signaling activation [61].

Finally, to confirm the prognostic relevance of the data, we took advantage of gene expression profiling (Affymetrix arrays) on the ex vivo collection of primary PCa cultures $(n=40$, Prognostic Marker Evaluation, Fig. 3A) from patients with 10-year documented clinical follow-up $[65,66]$. Gene panels enriched in primary cells derived from recurrent and non-recurrent cases were assigned either bad or good prognosis, respectively (Fig. S11A). Surprisingly and in line with our RPPA results, cells obtained from patients with poor prognosis displayed mRNA upregulation for the genes corresponding to significant prognostic RPPA candidates, i.e. TGFB1, WNT5A/B, SHC1, RAF1 and MYC (Fig. S11A, B). Western blotting analysis confirmed the over-expression of candidates in cells deriving from cases with poor prognosis (Fig. S11C). Overall, these data corroborate the hypothesis that an EV-based protein signature may have a clinically valuable prognostic significance.

\section{DISCUSSION}

Shreds of evidence have shown that EVs are an important source of cancer biomarkers [67]. Since for many cancers the amount of vesicles circulating in patients' biological fluids is acceptedly increased as compared to healthy individuals, the analysis of EV proteomic patterns [25] may provide a valuable strategy to complement current liquid-biopsy technologies, such as circulating tumor cells or DNA (ctDNA) analysis.

Here, we used the RPPA platform for multiplexed, highthroughput analysis of protein cargo in cancer-derived EVs. We first tested the sensitivity and specificity of this approach for the detection of selected proteins in EVs isolated from commercially available cancer cell lines. In particular, EpCAM expression was analyzed in EVs by RPPA assay and furtherly validated by immunogold electron microscopy and Western blotting.

Commercially available ELISA assays for EVs are based on EV immune-capture or lysate analysis $[27,68]$. Here, we devised a new homemade ELISA assay, i.e. ELEXO, as both an additional validation platform and a potential alternative to RPPA for rapid translation to the clinics of a low-throughput quantification assay for surface EV antigens.

To date, blood PSA screening is the sole non-invasive test for PCa diagnosis. In addition, among patients with elevated serum PSA levels ( $\geq 3-4 \mathrm{ng} / \mathrm{mL}$ ) and undergoing a standard transrectal ultrasound-guided biopsy, the chance of detecting $\mathrm{PCa}$ is approximately $30-40 \%$ [69]. Consequently, there is an urgent need for fast and multiparametric diagnostic tests based on non-invasive methods and requiring low amounts of patients' sample. In this regard, we conceived a pilot study on EVs isolated from a small cohort of PCas and found that a proteomic repertoire of as low as 37 antigens allows for the discrimination of tumors from healthy individuals. Interestingly, among the differentially expressed antigens found by us, IL- 6 has already been described as a tumor-discriminating antigen in PCa [70], while high levels of PD-L1, Integrin- $\beta 5$ and Survivin upregulation seems to correlate with cancer survival and aggressiveness [50, 51, 71, 72]. Subsequently, we extended the proteomic analysis to EVs isolated from a larger PCa cohort (training) including healthy donors and hypertrophic patients as control groups as well as post-prostatectomy disease-free patients as an additional comparative group. Again, based on the sole analysis of EV content, we found a proteomic signature capable of distinguishing tumors from controls. Of note, the set of differentially expressed endpoints include key players in cancer signaling networks. In particular, phosphorylation of $\beta$-catenin at T41-S45 determines its nuclear, transactivating activity [73], TSC2 phosphorylation (Y1571) associates with mTOR signaling activation [58, 74] and, intriguingly, the latter is coupled to low phospho-c-Myc_T58/S62, i.e. reduced c-Myc degradation rates $[75,76]$. The cartoon depicted in Fig. S7B exemplifies an hypothetical aberrant network flow, whereby i) an increased inhibitory phosphorylation of phospho-TSC2_Y1571 activates mTORC1 signaling, ii) augmented phospho-c-Abl_T735, directly relating to its sequestration into the cytoplasm by $14-3-3$ proteins, promotes TGF- $\beta$ activity [77] and iii) SEK1 phosphorylation favors metastatic spreading.

While pTNM and Gleason score are critical parameters to establish the risk of recurrence [78], their prognostic value remains poor leaving a clinical gap in the identification of PCas that have an intrinsic tendency to progress. Therefore, we studied a retrospective cohort, with 15 years of documented follow-up, to identify EV proteins indicative of tumor aggressiveness. Patients from this cohort were stratified into low/ intermediate- and high-risk cases and were analyzed together with a group of advanced tumors. Intriguingly, ERG overexpression correlated with advanced PCa forms and high-risk cases as well as with recurrence. High-risk and advanced PCas shared the expression of diverse proteins that are reportedly associated with cancer progression, namely TGF- $\beta$ [79], Wnt5a/b 
[34, 35, 80], Shc1 (phospho-SHCY317) [28], phospho-c-RAF-S338 and phospho-c-MycT58/S62 [81]. Moreover, we validated our results by measuring the expression of corresponding candidate genes on a collection of patient-derived immortalized cells [66] and confirmed the correlation between increased mRNA levels and a documented poor prognosis.

Since the management of PCa patients is currently under heated debate $[82,83]$, our predictive molecular signature may provide the opportunity to discriminate risk categories, improve the accuracy and feasibility of early diagnosis and suggest a potential monitoring method for suitable assignment of active surveillance protocols, nerve-sparing conservative surgery as well as radical treatments.

Although our data deserve further validation in dedicated, independent studies, the integration of the proteomic analysis of EVs with other available clinical and molecular parameters may lead to next-generation, multiparametric liquid-biopsy assays, thus providing a novel tool for screening, diagnosis, and risk assessment of PCa as well as of other cancers.

\section{MATERIALS AND METHODS Patient recruitment}

The study design is outlined in Fig. $3 \mathrm{~A}$ and clinical data were reported in Table S1. More specifically, a pivotal cohort composed of 12 primary prostate tumors and 16 healthy donors were used as technical proof of principle. The training cohort was composed of (i) 57 diagnosed primary tumors with 3-year clinically documented follow-up, (ii) 41 hypertrophic patients, (iii) 44 cases enrolled at least 12 months follow-up after prostatectomy and negative for recurrence or residual disease, and (iv) 18 age-matched healthy donors. A retrospective, risk assessment cohort (15-year follow-up; Table S5) including 19 tumor patients for prognostic marker estimation as well as 7 recurrent and 11 non-recurrent patients (and one additional patient with non-documented follow-up).

PCa patient stratification was performed by considering D'Amico risk categories [84], and ISUP/WHO 2016 classification, but arbitrarily stratifying cohorts as follows: Low risk: Gleason $=6$ and pTNM $\leq$ T2a; Low/Intermediate risk: Gleason $\leq 7(3+4)$ and pTNM $=\mathrm{T} 2 \mathrm{~b}-2 \mathrm{c} ;$ High risk: Gleason $=7(4+3)$ and $\mathrm{pTNM}=\mathrm{T} 2 \mathrm{c}$ and all Gleason score cases with pTNM $\geq \mathrm{T} 3 \mathrm{a}$. Cohorts were enrolled before COVID-19 pandemic. [pTNM: pathological TumorNode-Metastasis (pTNM). The NSC-lung cancer and colorectal tumor cohort clinical data were reported in Table S1.

\section{EV separation}

EV isolation was optimized as previously described [28] and following the MISEV2018 guidelines [30] Cell lines were cultivated in recommended medium and FBS (Fetal bovine serum) starved before EV concentration and processed with round of centrifugation as follows. Cell conditioned-starved medium was centrifuged 5 minutes at $1200 \mathrm{rpm}$ after $72 \mathrm{~h}$. The supernatants transferred in new tubes and centrifuged again for 15 minutes at $13300 \mathrm{rpm}$. Supernatants were transferred in clean tubes and ultracentrifuged (Beckman Coulter, Brea, CA) $2 \mathrm{~h}$ at $110,000 \mathrm{~g}$ (RCF). All centrifugation steps were performed at $4{ }^{\circ} \mathrm{C}$ temperature. (Beckman Coulter OptimaLE-80K-Ultracentrifuge; Rotor: BECKMAN SW41). Pellets were washed once and resuspended in $40 \mu \mathrm{l}$ of phosphate buffer saline (PBS). The concentration of EV suspension was measured by Bradford assay. $5 \mathrm{ml}$ of peripheral blood from participants were collected in serum clinical tubes (Code-367955-DBdiagnostics). Sera were collected and centrifuged 5 minutes at $1200 \mathrm{rpm}$ for serum separation and stored at $-80^{\circ} \mathrm{C} .1 \mathrm{ml}$ of serum for patient was used to obtain about 100-300 total $\mu \mathrm{g}$ of EVs. General patient exclusion criteria: patients with blood viral infection and different kind of tumors. For EVs separation, sera were thawed, transferred in clean tubes and diluted in PBS to reach equal volume of $1.4 \mathrm{ml}$. They were centrifuged 5 minutes at $1200 \mathrm{rpm}$, the supernatants transferred in new tubes and centrifuged again for 15 minutes at $13,300 \mathrm{rpm}$. Supernatants were transferred in clean tubes and ultracentrifuged (Beckman Coulter, Brea, CA) $2 \mathrm{~h}$ at $160,000 \mathrm{~g}$ (RCF). All centrifugation steps were performed at $4{ }^{\circ} \mathrm{C}$ temperature. (Sorvall-Ultracentrifige WX90-Ultra series, SN 42071706, ASHI; Rotor F50L-24×1.5). Pellets were washed once and resuspended in $40 \mu \mathrm{l}$ of phosphate buffer saline (PBS). The concentration of $\mathrm{EV}$ suspension was measured by Bradford assay.

\section{Reverse-phase Protein microArrays}

Reverse-Phase Protein microArrays (RPPA) were already optimized $[28,85]$. Briefly, all PBS-resuspended EV samples were lysed in a TPER (Thermo Fisher Scientific)-based lysis buffer for 30 minutes on ice. EV lysates were properly diluted and then printed in triplicate spots on nitrocellulose-coated glass slides (GRACE Bio-Labs, Bend, OR) using an Aushon 2470 equipped with $185 \mu \mathrm{m}$ pins (Aushon Biosystems, Billerica, $M A)$, according to the manufacturer's instructions at a concentration of $0.5 \mathrm{mg} / \mathrm{ml}$ and $0.125 \mathrm{mg} / \mathrm{ml}$. Reference standard lysates, comprised of HeLa + Pervanadate (BD, Franklin Lakes, NJ), Jurkat + Etoposide (Cell Signaling, Danvers, MA), Jurkat + Calyculin A (Cell Signaling), A431 + Pervanadate (Santa Cruz Biotechnologies) and A431 + EGF (BD, Franklin Lakes, $\mathrm{NJ}$ ) were printed in 10-point dilution curves as procedural controls and positive controls for antibody staining. Each reference was printed in triplicate at concentrations of $0.5 \mathrm{mg} / \mathrm{ml}$ and $0.125 \mathrm{mg} / \mathrm{ml}$. Per-spot protein concentration was evaluated by Sypro Ruby Protein Blot Stain (Invitrogen, Carlsbad, CA). Immediately prior to antibody staining, printed slides were treated with $1 \times$ ReBlot Mild Solution (Merck Millipore, Darmstadt, Germany) for $15 \mathrm{~min}$, washed $2 \times 5$ min with PBS (Invitrogen) and incubated for $2 \mathrm{~h}$ in blocking solution (2\% I-Block, (Applied Biosystems, Foster City, CA), $0.1 \%$ Tween-20 in PBS). Immunostaining was carried out using an avidin-biotin complex $(A B C)$ signal amplification kit (DAKO, Carpinteria, CA). Primary antibody binding was detected using a biotinylated goat anti-rabbit IgG H $+\mathrm{L}(1: 7500)$ (Vector Laboratories, Burlingame, CA) or rabbit anti-mouse IgG (1:10) (DAKO) followed by biotin amplification and streptavidin-conjugated IRDye680LT fluorophore (LI-COR Biosciences, Lincoln, NE). Primary antibodies against total and phosphorylated protein targets, were previously validated for singleband specificity by Western Blot using cell lysates (see Supplementary File 1). Negative control slides were incubated with secondary antibody alone. All Sypro and immunostained slides were scanned using a Tecan Power Scanner ${ }^{\mathrm{TM}}$ (Tecan Group Ltd, Switzerland). Acquired images were analyzed by MicroVigene v5.2 (VigeneTech, Carlisle, MA) software for spot detection, local background subtraction, negative control subtraction, replicate averaging, and total protein normalization. Throughout the manuscript and in the figures, the above-described normalized RPPA data are referred to as normalized RPPA intensity or levels and are expressed in arbitrary units (A.U.).

\section{Electron microscopy}

For Scanning Electron Microscopy (SEM) analysis, purified EVs were left to adhere to polylysine treated round glass coverslips $(10 \mathrm{~mm})$ for $4 \mathrm{~h}$ at RT and then fixed with glutaraldehyde $2,5 \%$ in sodium cacodylate buffer $0.1 \mathrm{M}$ overnight at $4{ }^{\circ} \mathrm{C}$. Samples were washed, post-fixed with $1 \%$ OsO4 in $0.1 \mathrm{M}$ sodium cacodylate buffer for $1 \mathrm{~h}$ at RT and dehydrated through a graded series of ethanol solutions (from $30 \%$ to $100 \%$ ). Ethanol was gradually substituted by a 1:1 solution of hexamethyldisilazane (HMDS) and absolute ethanol for $30 \mathrm{~min}$, successively by pure HMDS for $1 \mathrm{~h}$ (RT). Samples were completely dried by removing the HMDS and leaving at RT for $2 \mathrm{~h}$. Dried samples were mounted on stubs, coated with gold $(20 \mathrm{~nm})$ and analyzed in a FE-SEM Quanta Inspect F (FEl, Thermo Fisher Scientific). (Shively and Miller, 2009).

For Transmission Electron Microscopy (TEM) purified EVs from cellconditioned supernatants or serum were analyzed by negative staining method according to $[86,87]$ with slight modifications. Briefly, samples were let to adsorb on 400 mesh carbon-coated grids for $10 \mathrm{~min}$, blotted by filter paper and air dried. An ammonium molybdate $4 \%$ and phosphotungstic acid $2 \%(50 \% \mathrm{v} / \mathrm{v})$ contrasting solution $(\mathrm{pH} 6.8)$ was added for $20 \mathrm{~s}$ to each sample and blotted by filter paper. Samples were examined at $100 \mathrm{kV}$ by Philips EM208S TEM (FEI - Thermo Fisher Scientific), equipped with the Megaview II SIS camera (Olympus).

EV TEM images were analyzed by iTEM software to collect the diameter size of more than 100 vesicles for each sample. Only vesicles more than $20 \mathrm{~nm}$ in diameter were taken into account and the EV size distributions were expressed in a box plot.

Immuno-Electron Microscopy (IEM) was performed on extracellular vesicles adsorbed on carbon coated grids prepared as previously described for TEM negative staining. Grids were floated side down on a drop of PBS buffer and successively transferred on a drop of anti-EpCAM monoclonal antibody (Santa Cruz) (1:5 in PBS) over night at $4^{\circ} \mathrm{C}$. Then, samples were rinsed on PBS drops and incubated on $10 \mathrm{~nm}$ goldconjugated goat anti-mouse IgG serum (SIGMA) $(1: 30)$ for another $2 \mathrm{~h}$. Finally they were rinsed and stained by an ammonium molybdate $4 \%(\mathrm{pH}$ $6,8)$ and phpsphotungstic acid $2 \%$ solution $(50 \% \mathrm{v} / \mathrm{v})$. The excess fluid 
was blotted by filter paper and grids were air dried and observed Philips EM208S TEM (El -ThermoFisher).

\section{Cytofluorimetric and Western blot assay}

Flow cytometric analysis. Cancer cell lines (100,000 cells/tube) were incubated with antibodies against EpCAM (Miltenyi, Biotec, Custom antibody) and PD-L1 (XP version, Cell Signaling Danvers, MA) for $1 \mathrm{~h}$ on ice. Anti-lgG1 antibody was used as negative control (Abcam, Cambridge, UK). After 3 washes in PBS, samples were incubated with PE- or FITCconjugated secondary antibodies for $1 \mathrm{~h}$ on ice. FACS analysis was performed by FACS-Canto instrument (BD, Becton-Dickinson, Franklin Lakes, NJ, USA).

Western blotting. EVs were resuspended in RIPA lysis buffer and incubated for 15 minutes in ice. TPER (Thermo Fisher Scientific)-based lysis was used for sera EVs. Samples were centrifuged at $16,000 \times \mathrm{g}$ and supernatants were collected and transferred in a clean tube. After Bradford assay, EV lysates were diluted in LDS sample buffer (Thermofisher Scientific, Massachusetts, USA) at total amount of $40 \mu \mathrm{g}$. Samples were loaded and run into a 4-12\% polyacrylamide gel (Thermofisher Scientific, Massachusetts, USA) and transferred on nitrocellulose filters (SDS-PAGE) that were subsequently incubated with anti-EpCAM and anti-CD81 (Santa Cruz Biotechnology, California, USA) antibodies at $4{ }^{\circ} \mathrm{C}$ overnight. TSG101 and CD81 antibodies were used following relative datasheets Abcam and Santa Cruz. Then, filters were incubated with HRP-conjugated secondary anti-mouse or rabbit antibody (Southern Biotech, Birmingham, USA) for $1 \mathrm{~h}$ and signals detected with ECL method (GE Healthcare, Piscataway Township, NJ) by Chemidoc XRS system (Bio-Rad, Hercules, CA).

\section{Homemade ELISA 'ELEXO'}

EVs were resuspended in PBS (50 $\mathrm{\mu l}$ ) and deposited in a 96well-plate (Nunc, Milan, IT) at final concentration of $10 \mu \mathrm{g} /$ well and incubated for coating for $2 \mathrm{~h}$ at $37^{\circ} \mathrm{C}$ (or over-night). After the coating, three washes in PBS were performed. Coated plate was treated with blocking solution [PBS with $0.5 \%$ BSA (Bovine Serum Albumin pure protein); the solution was filtered with $0.200 \mu \mathrm{m}$ pores] for $2 \mathrm{~h}$. Primary Antibody staining was performed in PBS solution and incubated for $1 \mathrm{~h}$ at room temperature while secondary antibody (anti-mouse-HRP-conjugated antibody; Southern Biotech, Birmingham, USA) was diluted in PBS $/ 0.5 \%$ BSA solution for $1 \mathrm{~h}$ at room temperature. Anti-PD-L1 (86744-Cell Signaling Technology, Danvers, MA), anti-lgG1 (Abcam, Cambridge, UK), anti-CD81 Mab and anti-EpCAM Mab (Miltenyi Biotec, USA; Custom antibody) were used at final concentration of $2 \mu \mathrm{g} / \mathrm{ml}$. The plate was washed 3 times in PBS after each antibody incubation. After washing, the color-developing reaction with TMB (Thermoscientific, Massachusetts, USA) was started and then stopped with H2SO4. Optical densities were measured at $450 \mathrm{~nm}$ by Victor X3 (Perkin Elmer, Massachussets, USA). The blocking solution and the working antibody concentrations were optimized to minimize background staining with a relative scalar dilution of the reagents (i.e. BSA, primary and secondary antibodies).

\section{Luminex assay}

$100 \mu \mathrm{g}$ of EVs were lysed in $50 \mu \mathrm{l}$ of standard RIPA buffer [(20 mM Tris$\mathrm{HCl}$ pH7.2150 mM NaCl;1\% NP40 (Igepal CA-630); Distilled water to volume; Proteases-inhibitors)] and diluted 1:4 in PBS for the analysis. $100 \mu \mathrm{g}$ of parental EVs were left, non-lysed (SN), in $50 \mu \mathrm{l}$ in the buffer (PBS) of the last step of Ultracentrifugation and was directly analyzed by Luminex. Cytokine/chemokine quantification in EV extracts and in EV (SN) was achieved by xMAP technology through a Luminex platform (Bio-Rad Laboratories, Hercules, CA, USA) equipped with a magnetic washer workstation according to the manufacturer's protocol. RIPA (dilute 1:4 in PBS) and PBS buffer were used as background controls. Samples were analyzed using a human magnetic Luminex assay (R\&D Systems, Minneapolis, MN, USA). Brain-Derived Neutrophil Factor (BDNF), CCL11, Fibroblast Growth Factor 13 (FGF-13), IL-5, IL-4, IL-23, IL-6, MMP-2 (membrane-matrix-metalloprotease-2), beta-Nerve Growth Factor (beta-NGF), N-regulin-1 beta1/NRG-1, Tumor Necrosis Factor alpha (TNF-a), Interferon gamma-induced protein 10 (CXCL10), Interferon gamma (IFN- $\gamma)$, IL-2, IL-8/CXCL8, IL-17/IL-17A, CCL-2/MCP-1 and Vascular Endothelial Growth Factor (VEGF) were analyzed. The quantification was carried out with a Bio-Plex array reader (Bio-Plex 200 System) and Bio-Plex Manager (Version 6.1 Bio-Rad Laboratories, Hercules, CA, USA) software.

\section{Statistical analysis and data representation}

Protein analyses in EV samples. Student's t or non-parametric Wilcoxon rank-sum tests were used for continuous variables to analyze the differences between groups. A p-value $\leq 0.05$ was considered statistically significant. Furthermore, the receiver operating characteristic (ROC) method was used in order to find possible optimal cut-offs of the biomarkers capable of splitting patients into groups with different outcomes probabilities. Statistical analyses were conducted independently by means of SPSS ${ }^{\circledR}$ (v21.0) and MedCalc ${ }^{\circledR}(\mathrm{v} 10.0 .1)$ or ' $\mathrm{R}^{\prime}$ [88]. Data standardization (scaling), followed by two-way hierarchical clustering (Euclidean distance and Ward's method was used if not specified elsewhere), were performed by means of JMP v11 (SAS Institute, Cary, $N C)$ or ' $R$ ' [88] and RStudio [89]. Principal component analysis (PCA) as well as most data represented throughout the manuscript was independently reproduced by means of ' $R$ ' using the following packages: base, methods, utils, stats, graphics, grDevices, tcltk, openxlsx, tidyverse [90], data.table, RColorBrewer, reshape2, reshape, readxl, FactoMineR, factoextra, grid, gridExtra, circlize, cluster, dendextend and ComplexHeatmap [91].

Analysis of publicly available datasets. Messenger RNA results from Taylor's tissue dataset (NCBI GEO accession code GSE21032) have been accessed through the Prostate Cancer Genomics Data Portal (http:// cbioportal/) and combined with reported clinical data. Wilcoxon/Kruskal Wallis was used to analyze the differences between groups. GraphPad Prism v4 and JMP v11 (SAS Institute, Cary, NC) were used to perform statistical analyses.

\section{Cell lines, PCa-derived cells and gene expression profiling (Affymetrix)}

Cell cultures. All cell lines were obtained by ATCC. All cells were used as precocious (six passages) frozen stocks after arrival. They are routinely tested for Mycoplasma contamination ("PCR mycoplasma test kit", product no. A3744, PanReac AppliChem) before EV preparations. H1299, HT1975, HT29 cells were cultivated as recommended protocols. SW480 line was maintained in RPMI and 10\% of Fetal Bovine Serum (FBS). A431 and 293T cells were cultivated in DMEM (Dulbecco's Modified Eagle's Medium) with $10 \%$ of FBS, Glutamine (Gln) and Penicillin-Streptomycin (P/S) at standard doses. 293T cells were stable transduced with TWEEN vector [92] empty (Control) or with PD-L1 gene. Sequence-verified CDNA encoding for human PD-L1 was purchased from Dharmacon, cut with Xbal and EcoRV and inserted into TWEEN vector. It was kindly provided by Dr.Valeria Coppola. (PD-L1 Human-MGC Human CD274 Sequence-Verified CDNAClone ID: 30915301-Catalog Number: MHS6278-202856825)

(Lentiviral manipulation authorized by Ministry of Health rules. RM/IC/ Op2/17/002.notifica I.5.i.s/2017/15 - Biotecnologie. D.L.vo 206/2001). PC20 cancer activated fibroblast were obtained by tumor primary tissue cultures as by previously published [93].

PCa-derived cells. Primary PCa cultures were derived from freshlyexplanted tissue specimens (PCa-derived ex vivo model) following immortalization and phenotypic characterization. Clinical data and outcome of patients were collected for 15 years [66]. Briefly, poor prognosis group of donor patients with clinically localized PCa was defined by the presence of biochemical/local recurrence, metastasis, or disease-specific mortality, while the good prognosis group was defined by complete remission after surgery alone. Prognostic signature "Bad versus Good Prognosis profiling" was obtained in PCa cells by Affymetrix array (Human U133A Gene ChIP platform) using PCa cells derived from patients with different progression of disease (recurrent versus non-recurrent disease). Regulated biological processes were identified by the GOAL Web-based application and Gene Ontology (GO) terms with $p<0.01$ considered differentially regulated (false discovery rate $=0.013$ ).

Affymetrix data analysis. Affymetrix Gene Chip scanning was analyzed by customized R language-based script [88] using Bioconductor (http://www. bioconductor.org) for quality-control analysis, data normalization, hierarchical clustering, and identification of differentially expressed transcripts. Biological processes and molecular functions involved were identified by the GOAL Web-based application and the Unigene Build 154 according to the Gene Ontology (GO; http://www.geneontology.org) Consortium classification. Genes reported with $p<0.01$ were considered differentially regulated (false discovery rate $=0.013[65,66]$ ). 


\section{REFERENCES}

1. Bray F, Ferlay J, Soerjomataram I, Siegel RL, Torre LA, Jemal A. Global cancer statistics 2018: GLOBOCAN estimates of incidence and mortality worldwide for 36 cancers in 185 countries. CA: Cancer J. Clin. 2018;68:394-424.

2. De Angelis R, Sant M, Coleman MP, Francisci S, Baili P, Pierannunzio D, et al. Cancer survival in Europe 1999-2007 by country and age: results of EUROCARE-5a population-based study. Lancet Oncol. 2014;15:23-34.

3. Walters S, Maringe C, Coleman MP, Peake MD, Butler J, Young N, et al. Lung cancer survival and stage at diagnosis in Australia, Canada, Denmark, Norway, Sweden and the UK: a population-based study, 2004-2007. Thorax 2013;68:551-64.

4. Neppl-Huber C, Zappa M, Coebergh JW, Rapiti E, Rachtan J, Holleczek B, et al. Changes in incidence, survival and mortality of prostate cancer in Europe and the United States in the PSA era: additional diagnoses and avoided deaths. Ann Oncol. 2012;23:1325-34.

5. O'Brien K, Breyne K, Ughetto S, Laurent LC, Breakefield XO. RNA delivery by extracellular vesicles in mammalian cells and its applications. Nat Rev Mol Cell Biol. 2020;21:585-606.

6. Xu R, Rai A, Chen M, Suwakulsiri W, Greening DW, Simpson RJ. Extracellular vesicles in cancer-implications for future improvements in cancer care. Nat Rev Clin Oncol. 2018;15:617-38.

7. Li SP, Lin ZX, Jiang XY, Yu XY. Exosomal cargo-loading and synthetic exosomemimics as potential therapeutic tools. Acta Pharmacologica Sin. 2018;39:542-51.

8. Logozzi M, De Milito A, Lugini L, Borghi M, Calabrò L, Spada M, et al. High levels of exosomes expressing CD63 and caveolin-1 in plasma of melanoma patients. PloS ONE. 2009;4:e5219.

9. Sharma P, Diergaarde B, Ferrone S, Kirkwood JM, Whiteside TL. Melanoma cellderived exosomes in plasma of melanoma patients suppress functions of immune effector cells. Sci. Rep. 2020;10:92.

10. Whiteside TL. The potential of tumor-derived exosomes for noninvasive cancer monitoring: an update. Expert Rev. Mol. Diagn. 2018;18:1029-40.

11. Alfonsi R, Grassi L, Signore M, Bonci D. The double face of exosome-carried MicroRNAs in cancer immunomodulation. Int J Mol Sci. 2018;19:1183.

12. Liu Y, Cao X. Organotropic metastasis: role of tumor exosomes. Cell Res. 2016;26:149-50.

13. Poggio M, Hu T, Pai CC, Chu B, Belair CD, Chang A, et al. Suppression of exosomal PDL1 induces systemic anti-tumor immunity and memory. Cell 2019;177:414-27. e13.

14. Rajagopal C, Harikumar KB. The origin and functions of exosomes in cancer. Front Oncol. 2018;8:66.

15. Fevrier B, Vilette D, Archer F, Loew D, Faigle W, Vidal M, et al. Cells release prions in association with exosomes. Proc Natl Acad Sci USA. 2004;101:9683-8.

16. Asare-Werehene M, Nakka K, Reunov A, Chiu CT, Lee WT, Abedini MR, et al. The exosome-mediated autocrine and paracrine actions of plasma gelsolin in ovarian cancer chemoresistance. Oncogene 2020;39:1600-16.

17. Meldolesi J. Exosomes and ectosomes in intercellular communication. Curr Biol: CB. 2018;28:R435-r44.

18. Tang X, Chang C, Guo J, Lincoln V, Liang C, Chen M, et al. Tumour-secreted Hsp90alpha on external surface of exosomes mediates tumour-stromal cell communication via autocrine and paracrine mechanisms. Sci. Rep. 2019;9:15108.

19. Costa-Silva B, Aiello NM, Ocean AJ, Singh S, Zhang H, Thakur BK, et al. Pancreatic cancer exosomes initiate pre-metastatic niche formation in the liver. Nat Cell Biol. 2015; 17:816-26.

20. Hoshino A, Costa-Silva B, Shen TL, Rodrigues G, Hashimoto A, Tesic Mark M, et al. Tumour exosome integrins determine organotropic metastasis. Nature 2015;527:329-35.

21. Taylor BS, Schultz N, Hieronymus H, Gopalan A, Xiao Y, Carver BS, et al. Integrative genomic profiling of human prostate cancer. Cancer Cell. 2010;18:11-22.

22. Hussain M, Mateo J, Fizazi K, Saad F, Shore N, Sandhu S, et al. Survival with olaparib in metastatic castration-resistant prostate cancer. $\mathrm{N}$ Engl J Med. 2020;383:2345-57.

23. Zheng SL, Sun J, Wiklund F, Smith S, Stattin P, Li G, et al. Cumulative association of five genetic variants with prostate cancer. N. Engl. J. Med. 2008;358:910-9.

24. Lorenc T, Klimczyk K, Michalczewska I, Slomka M, Kubiak-Tomaszewska G, Olejarz W. Exosomes in prostate cancer diagnosis, prognosis and therapy. Int J Mol Sci. 2020;21:2118.

25. Flores-Morales A, Iglesias-Gato D. Quantitative mass spectrometry-based proteomic profiling for precision medicine in prostate cancer. Front Oncol. 2017;7:267.

26. Drake JM, Paull EO, Graham NA, Lee JK, Smith BA, Titz B, et al. Phosphoproteome integration reveals patient-specific networks in prostate. Cancer Cell. 2016;166:1041-54.

27. Malik A, Srinivasan S, Batra J. A new era of prostate cancer precision medicine. Front Oncol. 2019;9:1263.

28. Cannistraci A, Federici G, Addario A, Di Pace AL, Grassi L, Muto G, et al. C-Met/ miR-130b axis as novel mechanism and biomarker for castration resistance state acquisition. Oncogene 2017;36:3718-28.
29. Vinik Y, Ortega FG, Mills GB, Lu Y, Jurkowicz M, Halperin S, et al. Proteomic analysis of circulating extracellular vesicles identifies potential markers of breast cancer progression, recurrence, and response. Sci Adv. 2020;6:eaba5714.

30. Thery C, Witwer KW, Aikawa E, Alcaraz MJ, Anderson JD, Andriantsitohaina R, et al. Minimal information for studies of extracellular vesicles 2018 (MISEV2018): a position statement of the International Society for Extracellular Vesicles and update of the MISEV2014 guidelines. J Extracell Vesicles. 2018;7:1535750.

31. Jiang L, Shen Y, Guo D, Yang D, Liu J, Fei X, et al. EpCAM-dependent extracellular vesicles from intestinal epithelial cells maintain intestinal tract immune balance. Nat Commun. 2016;7:13045.

32. Munz M, Baeuerle PA, Gires $O$. The emerging role of EpCAM in cancer and stem cell signaling. Cancer Res. 2009;69:5627-9.

33. De Rubis G, Rajeev Krishnan S, Bebawy M. Liquid biopsies in cancer diagnosis, monitoring, and prognosis. Trends Pharm Sci. 2019;40:172-86.

34. Hase T, Sato M, Yoshida K, Girard L, Takeyama Y, Horio M, et al. Pivotal role of epithelial cell adhesion molecule in the survival of lung cancer cells. Cancer Sci. 2011;102:1493-500.

35. Manzotti G, Torricelli F, Benedetta D, Lococo F, Sancisi V, Rossi G, et al. An epithelial-to-mesenchymal transcriptional switch triggers evolution of pulmonary sarcomatoid carcinoma (PSC) and identifies dasatinib as new therapeutic option. Clin Cancer Res. 2019;25:2348-60.

36. Weiswald LB, Guinebretière JM, Richon S, Bellet D, Saubaméa B, Dangles-Marie V. In situ protein expression in tumour spheres: development of an immunostaining protocol for confocal microscopy. BMC Cancer. 2010;10:106.

37. Fattore L, Ruggiero CF, Liguoro D, Mancini R, Ciliberto G. Single cell analysis to dissect molecular heterogeneity and disease evolution in metastatic melanoma. Cell Death Dis. 2019;10:827.

38. Haigler H, Ash JF, Singer SJ, Cohen S. Visualization by fluorescence of the binding and internalization of epidermal growth factor in human carcinoma cells A-431. Proc Natl Acad Sci USA. 1978;75:3317-21.

39. Kaplan M, Narasimhan S, de Heus C, Mance D, van Doorn S, Houben K, et al. EGFR dynamics change during activation in native membranes as revealed by NMR. Cell 2016;167:1241-51. e11.

40. Kriegs M, Clauditz TS, Hoffer K, Bartels J, Buhs S, Gerull H, et al. Analyzing expression and phosphorylation of the EGF receptor in HNSCC. Sci. Rep. 2019;9:13564.

41. Horita H, Law A, Hong S, Middleton K. Identifying regulatory posttranslational modifications of PD-L1: a focus on monoubiquitinaton. Neoplasia (New York, NY). 2017;19:346-53.

42. Teramoto $K$, Igarashi T, Kataoka $Y$, Ishida M, Hanaoka J, Sumimoto $H$, et al. Clinical significance of PD-L1-positive cancer-associated fibroblasts in pNOMO non-small cell lung cancer. Lung Cancer (Amst., Neth.). 2019;137:56-63.

43. Ready N, Hellmann MD, Awad MM, Otterson GA, Gutierrez M, Gainor JF, et al. First-line nivolumab plus ipilimumab in advanced non-small-cell lung cancer (CheckMate 568): outcomes by programmed death ligand 1 and tumor mutational burden as biomarkers. J Clin Oncol. 2019;37:992-1000.

44. Linxweiler J, Junker K. Extracellular vesicles in urological malignancies: an update. Nat Rev Urol. 2020;17:11-27.

45. Kowal J, Arras G, Colombo M, Jouve M, Morath JP, Primdal-Bengtson B, et al. Proteomic comparison defines novel markers to characterize heterogeneous populations of extracellular vesicle subtypes. Proc Natl Acad Sci USA. 2016;113: E968-77.

46. Kalluri R, LeBleu VS. The biology, function, and biomedical applications of exosomes. Science. 2020;367:eaau6977.

47. Zhang $H$, Freitas D, Kim HS, Fabijanic K, Li Z, Chen $H$, et al. Identification of distinct nanoparticles and subsets of extracellular vesicles by asymmetric flow field-flow fractionation. Nat Cell Biol. 2018;20:332-43.

48. Li C, Li C, Zhi C, Liang W, Wang X, Chen X, et al. Clinical significance of PD-L1 expression in serum-derived exosomes in NSCLC patients. J Transl Med. 2019;17:355.

49. Tomlins SA, Day JR, Lonigro RJ, Hovelson DH, Siddiqui J, Kunju LP, et al. Urine TMPRSS2:ERG plus PCA3 for individualized prostate cancer risk assessment. Eur. Urol. 2016;70:45-53.

50. Gevensleben H, Dietrich D, Golletz C, Steiner S, Jung M, Thiesler T, et al. The immune checkpoint regulator PD-L1 is highly expressed in aggressive primary prostate cancer. Clin. Cancer Res. 2016;22:1969-77.

51. Li F, Aljahdali I, Ling X. Cancer therapeutics using survivin BIRC5 as a target: what can we do after over two decades of study? J Exp Clin Cancer Res. 2019;38:368.

52. Culig Z, Puhr M. Interleukin- 6 and prostate cancer: current developments and unsolved questions. Mol. Cell Endocrinol. 2018;462:25-30.

53. Le T, Gerber DE. Newer-generation EGFR inhibitors in lung cancer: how are they best used? Cancers. 2019;11:366.

54. Vaz J, Ansari D, Sasor A, Andersson R. SPARC: a potential prognostic and therapeutic target in pancreatic cancer. Pancreas 2015;44:1024-35. 
55. Michalaki V, Syrigos K, Charles P, Waxman J. Serum levels of IL-6 and TNF-alpha correlate with clinicopathological features and patient survival in patients with prostate cancer. Br J Cancer. 2004;90:2312-6.

56. Smolarz M, Pietrowska M, Matysiak N, Mielańczyk $Ł$, Widłak P. Proteome profiling of exosomes purified from a small amount of human serum: the problem of copurified serum components. Proteomes. 2019;7:18.

57. Tang Z, Li C, Kang B, Gao G, Li C, Zhang Z. GEPIA: a web server for cancer and normal gene expression profiling and interactive analyses. Nucleic Acids Res. 2017;45:W98-w102

58. Inoki K, Li Y, Zhu T, Wu J, Guan KL. TSC2 is phosphorylated and inhibited by Akt and suppresses mTOR signalling. Nat. Cell Biol. 2002;4:648-57.

59. Alonzo DG, Mure AL, Soloway MS. Prostate cancer and the increasing role of active surveillance. Postgrad Med. 2013;125:109-16.

60. Klotz L, Emberton M. Management of low risk prostate cancer-active surveillance and focal therapy. Nat Rev Clin Oncol. 2014;11:324-34.

61. Haider M, Zhang X, Coleman I, Ericson N, True LD, Lam HM, et al. Epithelial mesenchymal-like transition occurs in a subset of cells in castration resistant prostate cancer bone metastases. Clin. Exp. Metastasis. 2016;33:239-48.

62. Cai $\mathrm{C}$, Wang $\mathrm{H}$, He HH, Chen $\mathrm{S}$, He L, Ma F, et al. ERG induces androgen receptormediated regulation of $\mathrm{SOX} 9$ in prostate cancer. J Clin Investig. 2013;123:1109-22.

63. Ankerst DP, Goros M, Tomlins SA, Patil D, Feng Z, Wei JT, et al. Incorporation of urinary prostate cancer antigen 3 and TMPRSS2:ERG into prostate cancer prevention trial risk calculator. Eur Urol Focus. 2019;5:54-61.

64. Singhal U, Wang Y, Henderson J, Niknafs YS, Qiao Y, Gursky A, et al. Multigene profiling of CTCs in $\mathrm{mCRPC}$ identifies a clinically relevant prognostic signature. Mol Cancer Res: MCR. 2018;16:643-54.

65. Nanni S, Aiello A, Re A, Guffanti A, Benvenuti V, Colussi C, et al. Estrogendependent dynamic profile of eNOS-DNA associations in prostate cancer. PloS ONE. 2013;8:e62522.

66. Nanni S, Priolo C, Grasselli A, D'Eletto M, Merola R, Moretti F, et al. Epithelialrestricted gene profile of primary cultures from human prostate tumors: a molecular approach to predict clinical behavior of prostate cancer. Mol Cancer Res: MCR. 2006;4:79-92

67. LeBleu VS, Kalluri R. Exosomes as a multicomponent biomarker platform in cancer. Trends Cancer 2020;6:767-74.

68. Wang L, Skotland T, Berge V, Sandvig K, Llorente A. Exosomal proteins as prostate cancer biomarkers in urine: from mass spectrometry discovery to immunoassaybased validation. Eur. J. Pharm. Sci. 2017;98:80-5.

69. Ploussard G, Nicolaiew N, Marchand C, Terry S, Vacherot F, Vordos D, et al. Prospective evaluation of an extended 21-core biopsy scheme as initial prostate cancer diagnostic strategy. Eur. Urol. 2014;65:154-61.

70. Adler HL, McCurdy MA, Kattan MW, Timme TL, Scardino PT, Thompson TC. Elevated levels of circulating interleukin- 6 and transforming growth factor-beta1 in patients with metastatic prostatic carcinoma. J. Urol. 1999;161:182-7.

71. Pidgeon GP, Tang K, Cai YL, Piasentin E, Honn KV. Overexpression of platelet-type 12-lipoxygenase promotes tumor cell survival by enhancing alpha(v)beta(3) and alpha(v)beta(5) integrin expression. Cancer Res. 2003;63:4258-67.

72. Zhang LY, Guo Q, Guan GF, Cheng W, Cheng P, Wu AH. Integrin Beta 5 is a prognostic biomarker and potential therapeutic target in glioblastoma. Front Oncol. 2019;9:904

73. Liu C, Li Y, Semenov M, Han C, Baeg GH, Tan Y, et al. Control of beta-catenin phosphorylation/degradation by a dual-kinase mechanism. Cell 2002;108:837-47.

74. Aicher LD, Campbell JS, Yeung RS. Tuberin phosphorylation regulates its interaction with hamartin. Two proteins involved in tuberous sclerosis. J Biol Chem. 2001;276:21017-21.

75. Sears R, Nuckolls F, Haura E, Taya Y, Tamai K, Nevins JR. Multiple Ras-dependent phosphorylation pathways regulate Myc protein stability. Genes Dev. 2000;14:2501-14

76. Yeh E, Cunningham M, Arnold H, Chasse D, Monteith T, Ivaldi G, et al. A signalling pathway controlling c-Myc degradation that impacts oncogenic transformation of human cells. Nat Cell Biol. 2004:6:308-18.

77. Morrison CD, Parvani JG, Schiemann WP. The relevance of the TGF- $\beta$ Paradox to EMT-MET programs. Cancer Lett. 2013;341:30-40.

78. Lei JH, Liu LR, Wei Q, Yan SB, Song TR, Lin FS, et al. Systematic review and metaanalysis of the survival outcomes of first-line treatment options in high-risk prostate cancer. Sci Rep. 2015;5:7713.

79. Kang JH, Jung MY, Choudhury M, Leof EB. Transforming growth factor beta induces fibroblasts to express and release the immunomodulatory protein PD-L1 into extracellular vesicles. FASEB J. 2020;34:2213-26.

80. Bojkova D, Klann K, Koch B, Widera M, Krause D, Ciesek S, et al. Proteomics of SARS-CoV-2-infected host cells reveals therapy targets. Nature. 2020;583:469-72.

81. Reyna MA, Haan D, Paczkowska M, Verbeke LPC, Vazquez M, Kahraman A, et al. Pathway and network analysis of more than 2500 whole cancer genomes. Nat. Commun. 2020;11:729.
82. Klotz L. The future of active surveillance. Transl Androl Urol. 2018;7:256-9.

83. Klotz L. Active surveillance in intermediate-risk prostate cancer. BJU Int 2020;125:346-54

84. Slack FJ, Chinnaiyan AM. The role of non-coding RNAs in oncology. Cell 2019;179:1033-55.

85. Matteoni S, Abbruzzese C, Matarrese P, De Luca G, Mileo AM, Miccadei S, et al. The kinase inhibitor SI113 induces autophagy and synergizes with quinacrine in hindering the growth of human glioblastoma multiforme cells. J Exp Clin Cancer Res. 2019;38:202

86. Shively S, Miller WR. The use of HMDS (hexamethyldisilazane) to replace Critical Point Drying (CPD) in the preparation of tardigrade for SEM (Scanning Electron Microscope) imaging. Trans Kans Acad Sci. (1903). 2009;112: 198-200.

87. Thery C, Amigorena S, Raposo G, Clayton A. Isolation and characterization of exosomes from cell culture supernatants and biological fluids. Curr Protoc Cell Biol. 2006;Chapter 3:Unit 322.

88. R: A Language and Environment for Statistical Computing [Internet]. R Core Team. 2018. Available from: http://www.R-project.org/.

89. RStudio: Integrated Development Environment for R [Internet]. RStudio Team. 2020. Available from: http://www.rstudio.com/.

90. Wickham H, Averick M, Bryan J, Chang W, McGowan L, François R, et al. Welcome to the Tidyverse. J Open Source Softw. 2019;4:1686.

91. Gu Z, Eils R, Schlesner M. Complex heatmaps reveal patterns and correlations in multidimensional genomic data. Bioinform (Oxf, Engl). 2016;32:2847-9.

92. Bonci D, Coppola V, Musumeci M, Addario A, Giuffrida R, Memeo L, et al. The miR15a-miR-16-1 cluster controls prostate cancer by targeting multiple oncogenic activities. Nat Med. 2008;14:1271-7.

93. Musumeci M, Coppola V, Addario A, Patrizii M, Maugeri-Sacca M, Memeo L, et al Control of tumor and microenvironment cross-talk by miR-15a and miR-16 in prostate cancer. Oncogene 2011;30:4231-42.

94. Lex A, Gehlenborg N, Strobelt H, Vuillemot R, Pfister H. UpSet: visualization of intersecting sets. IEEE Trans. Vis. Comput Graph. 2014;20:1983-92.

\section{ACKNOWLEDGEMENTS}

The authors thank Giuseppe Loreto, Paola Di Matteo, Roberto Ricci, Laura De Salvo, Marco Varmi and Mustapha Haoui for their technical support. Claudio Tabolacci was supported by Fondazione Umberto Veronesi, which is gratefully acknowledged.

\section{AUTHOR CONTRIBUTIONS}

M.S., R.A., G.F., A.A.D. performed RPPA and Western blotting analysis and E.V. sample preparation; M.S. and G.F. performed database data analysis; S.N., A.A.I. executed primary culture experiment analysis; LBE achieved TEM, SEM, and IEM analyses; M.D. made TEM experiments; I.S. and M.S. performed statistical analysis; A.L.D.P. and L.B. realized E.V. sample preparation, FACS analysis, ELEXO optimization, and experiments; G.M., A.G., D.C., G.S., M.C., L.C., M.G., R.P. enrolled patient cohorts, collected samples, and clinical information databases, and promoted the trial clinical design; S.S. performed anatomic pathology evaluation of the enrolled cohorts; S.R., C.T. performed Luminex experiments; T.M. performed English language revision; M.S. compiled the figures; D.B., R.D.M. coordinated and proposed the trial; D.B., M.S., R.D. M. created the experimental design and clinical sets; D.B., M.S. and R.D.M. wrote the manuscript.

\section{FUNDING STATEMENTS}

The present study was supported by: National Ministry of Health, Under-forty researcher (GR:2011-02351557) to D.B., S.N., and A.G.; Oncotecnologico program 15ONC/5 to D.B.; Transcan-2-JTC 2016-Prolipsy and PON "BiLiGeCT" ARS01_00492 to D.B.

\section{ETHICS APPROVAL AND CONSENT TO PARTICIPATE}

All patients' samples were collected and handled in the study following the ethical internationally recognized guidelines and the project was approved by the Ethics Committees. Prospective training-like and advanced cancer pivotal cohorts were collected at San Giovanni Bosco and Humanitas, Turin hospitals and Regina Elena National Cancer Institute under the approval of Istituto Superiore di Sanità (CE-ISS 09250, 2009; Prot. CE-ISS-PRE 17/18 of 11/01/2018; CE-IRE-350-13). Retrospective prostate and colon adenocarcinoma cases were collected at Regina Elena National Cancer Institute under the Italy-USA program and subjected to approval by Istituto Superiore di Sanità (Prot-PRE 202/06-ref-CE-ISS 06/140). NSCL-cancer were collected under Ethics Committees approval (Prot-ISS-PRE-416/17). 
14

\section{COMPETING INTERESTS}

The authors declare no competing interests.

\section{ADDITIONAL INFORMATION}

Supplementary information The online version contains supplementary material available at https://doi.org/10.1038/s41419-021-03909-z.

Correspondence and requests for materials should be addressed to D.B.

Reprints and permission information is available at http://www.nature.com/ reprints

Publisher's note Springer Nature remains neutral with regard to jurisdictional claims in published maps and institutional affiliations.
Open Access This article is licensed under a Creative Commons Attribution 4.0 International License, which permits use, sharing, adaptation, distribution and reproduction in any medium or format, as long as you give appropriate credit to the original author(s) and the source, provide a link to the Creative Commons license, and indicate if changes were made. The images or other third party material in this article are included in the article's Creative Commons license, unless indicated otherwise in a credit line to the material. If material is not included in the article's Creative Commons license and your intended use is not permitted by statutory regulation or exceeds the permitted use, you will need to obtain permission directly from the copyright holder. To view a copy of this license, visit http://creativecommons. org/licenses/by/4.0/.

(c) The Author(s) 2021 